\title{
Effects of acute tryptophan depletion on memory, attention and executive functions: a systematic review
}

Citation for published version (APA):

Mendelsohn, D., Riedel, W. J., \& Sambeth, A. (2009). Effects of acute tryptophan depletion on memory, attention and executive functions: a systematic review. Neuroscience and Biobehavioral Reviews, 33(6), 926-952. https://doi.org/10.1016/j.neubiorev.2009.03.006

Document status and date:

Published: 01/01/2009

DOI:

10.1016/j.neubiorev.2009.03.006

Document Version:

Publisher's PDF, also known as Version of record

Document license:

Taverne

Please check the document version of this publication:

- A submitted manuscript is the version of the article upon submission and before peer-review. There can be important differences between the submitted version and the official published version of record.

People interested in the research are advised to contact the author for the final version of the publication, or visit the DOI to the publisher's website.

- The final author version and the galley proof are versions of the publication after peer review.

- The final published version features the final layout of the paper including the volume, issue and page numbers.

Link to publication

\footnotetext{
General rights rights.

- You may freely distribute the URL identifying the publication in the public portal. please follow below link for the End User Agreement:

www.umlib.nl/taverne-license

Take down policy

If you believe that this document breaches copyright please contact us at:

repository@maastrichtuniversity.nl

providing details and we will investigate your claim.
}

Copyright and moral rights for the publications made accessible in the public portal are retained by the authors and/or other copyright owners and it is a condition of accessing publications that users recognise and abide by the legal requirements associated with these

- Users may download and print one copy of any publication from the public portal for the purpose of private study or research.

- You may not further distribute the material or use it for any profit-making activity or commercial gain

If the publication is distributed under the terms of Article $25 \mathrm{fa}$ of the Dutch Copyright Act, indicated by the "Taverne" license above, 


\title{
Review
}

\section{Effects of acute tryptophan depletion on memory, attention and executive functions: A systematic review}

\author{
Daniel Mendelsohn ${ }^{\mathrm{a}, \mathrm{b}}$, Wim J. Riedel ${ }^{\mathrm{a}}$, Anke Sambeth ${ }^{\mathrm{a}, *}$

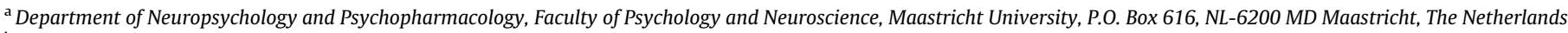 \\ ${ }^{\mathrm{b}}$ Schulich School of Medicine and Dentistry, The University of Western Ontario, London, Ontario, Canada
}

\section{A R T I C L E I N F O}

\section{Article history:}

Received 9 January 2009

Received in revised form 10 March 2009

Accepted 11 March 2009

\section{Keywords:}

Tryptophan depletion

Serotonin

Cognition

Memory

Attention

Executive function

\begin{abstract}
A B S T R A C T
The serotonergic system is implicated in the regulation of mood and cognition. Acute tryptophan depletion (ATD) is an experimental procedure for lowering central serotonin levels. Here, the effects of ATD on psychomotor processing, declarative memory, working memory, executive functions and attention are discussed. The most robust finding is that ATD impairs the consolidation of episodic memory for verbal information. Semantic memory appears to be unaffected by ATD although a limited variety of tasks examined effects in this domain. Similarly, evidence suggests ATD does not influence verbal, spatial and affective working memory. Most studies investigating effects on executive functions have produced non-specific or negative findings. In terms of attention, ATD either does not affect or may improve focused attention and ATD likely does not impact sustained and divided attention or attentional set-shifting. Although ATD is known to affect mood in certain vulnerable populations, the effects of ATD on cognition in non-vulnerable participants are independent of mood changes. Suggestions for future directions and implications for psychiatric illnesses are discussed.
\end{abstract}

(c) 2009 Elsevier Ltd. All rights reserved.

\section{Contents}

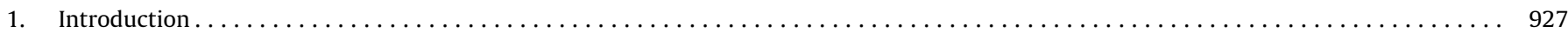

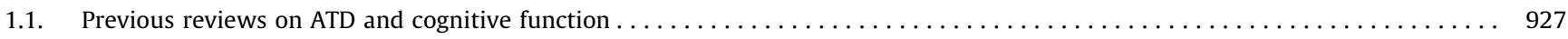

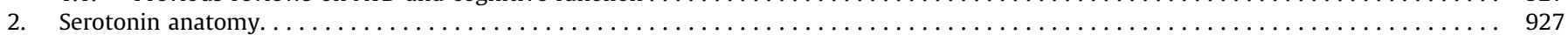

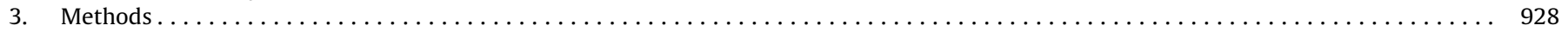

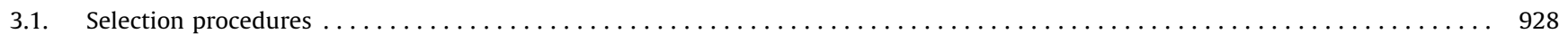

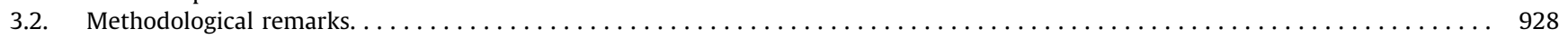

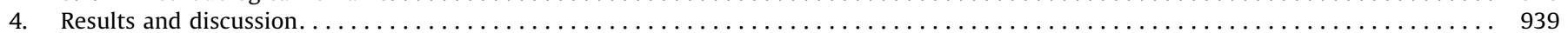

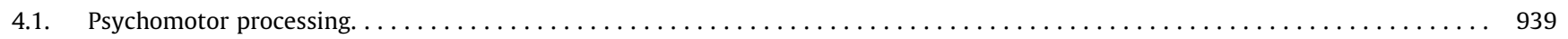

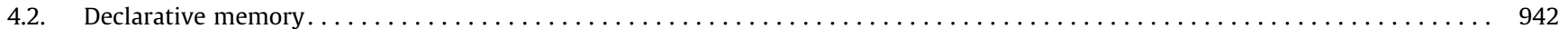

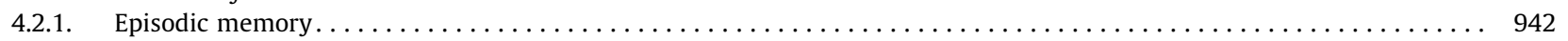

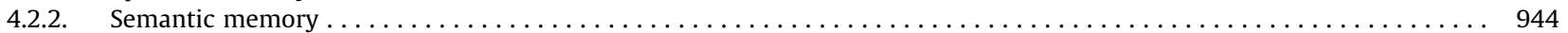

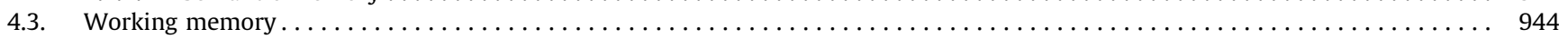

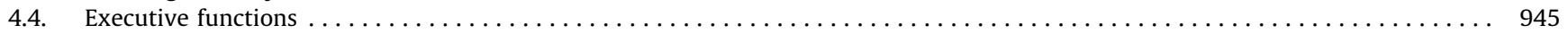

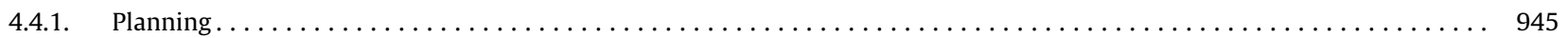

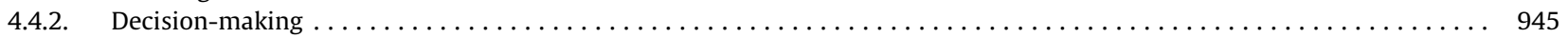

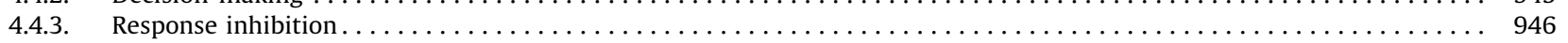

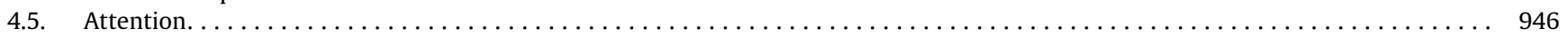

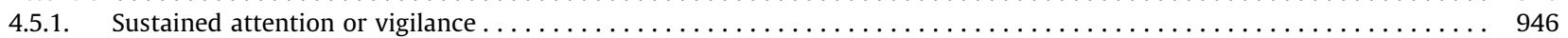

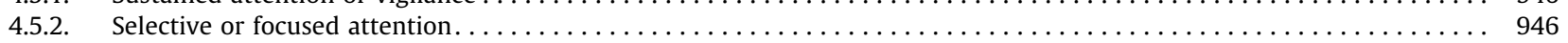

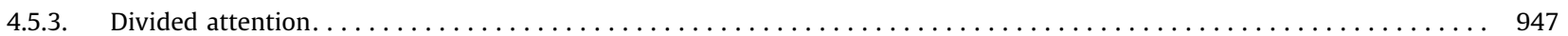

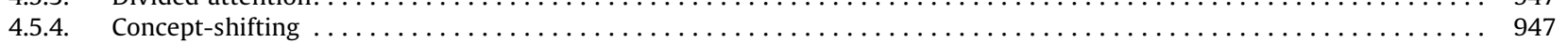

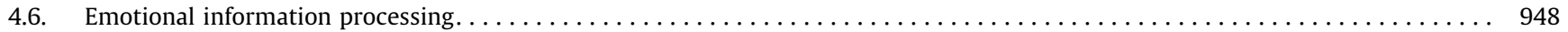

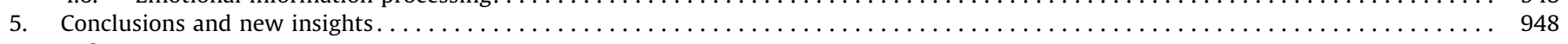

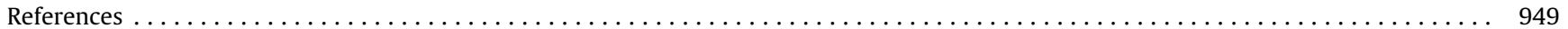

* Corresponding author. Tel.: +31 433881757; fax: +31 433884560

E-mail address: anke.sambeth@psychology.unimaas.nl (A. Sambeth). 


\section{Introduction}

Serotonin (5-hydroxytryptamine, 5-HT) is the neurotransmitter of a diffuse modulatory system implicated in mood regulation and cognition (Riedel, 2004; Schmitt et al., 2006). A number of psychiatric and mood disorders are related to serotonergic dysfunction including depression (Arango et al., 2002; Delgado, 2000), anxiety disorders (Deakin, 1998; Stein and Stahl, 2000), bipolar disorder (Mahmood and Silverstone, 2001), schizophrenia (Lee and Meltzer, 2001) and obsessive-compulsive disorder (OCD) (Zohar et al., 2004). Indeed medications that augment serotonergic activity are standard treatments for depression (Mace and Taylor, 2000) and anxiety disorders (Kent et al., 1998). Several of these psychiatric disorders are often accompanied by a cognitive impairment (Bearden et al., 2001; Elvevag and Goldberg, 2000; Porter et al., 2003a) that may be related to serotonergic dysfunction. Due to serotonin's significance in neuropathology and serotonin's potential role in cognitive dysfunction, research in this field has gained increasing attention in recent years.

Experiments involving pharmacological modulators of serotonergic activity are complicated by the existence of several types of 5-HT receptors that are differentially affected by serotoninmodulating agents (Barnes and Sharp, 1999; Buhot, 1997). As a result, the acute tryptophan depletion (ATD) technique for lowering whole-brain 5-HT levels has become increasingly popular. ATD is an experimental method for decreasing central serotonergic activity (Young et al., 1985). The ingestion of a solution containing large neutral amino acids that is deficient in Ltryptophan (TRP), the amino acid precursor necessary for 5-HT synthesis, induces a reliable and reversible lowering of specifically TRP concentrations in the blood (Boadle-Biber, 1993; Klaassen et al., 1999; Reilly et al., 1997; Smith et al., 1987; Van der Does, 2001). Central depletion is achieved by the combined effects of increased protein synthesis incorporating TRP in organs outside the central nervous system that reduces available precursor in plasma (Biggio et al., 1974), a decrease in the ratio of TRP to large neutral amino acids (Fernstrom and Faller, 1978), and competition between the large neutral amino acids and the precursor for transport into the central nervous system across the blood-brain barrier (Oldendorf and Szabo, 1976). A fundamental issue is the validity of the ATD method for lowering central 5-HT levels. Experiments in rats have shown that ATD did not only reduce TRP ratios in the blood plasma, but also in several brain regions such as the hippocampus and striatum (Lieben et al., 2004). Furthermore, 5 -HT levels in the hippocampus, striatum, and cortex were reduced by around 50\% (Blokland et al., 2002). ATD also reduces plasma and cerebrospinal fluid TRP levels and decreases brain 5-HT synthesis in humans (Nishizawa et al., 1997; Williams et al., 1999) and monkeys (Young et al., 1989). In humans, the ATD procedure is capable of decreasing plasma TRP by $45-90 \%$, reaching maximum plasma depletion after 5-7 h (Reilly et al., 1997; Van der Does, 2001). Therefore, ATD is a valid and effective method for lowering central 5-HT levels.

Since 5-HT plays a major role in depression, the effects of ATD on mood in humans have been explored extensively. In a seminal study, Young et al. (1985) reported that ATD increased selfreported ratings of depression in healthy volunteers; a finding replicated by the same group (Smith et al., 1987). Other studies found small effects on mood (Ellenbogen et al., 1996; Weltzin et al., 1994) or no effect (Abbott et al., 1992; Oldman et al., 1994; Weltzin et al., 1995) (see Van der Does, 2001; Booij et al., 2003 for a review). A recent meta-analysis concluded that ATD decreases mood in patients with remitted depression, patients with depression taking anti-depressants, and participants with family histories of depression but not in healthy controls without family histories of depression (Ruhe et al., 2007). Another pooled 'mega-analysis' identified similar risk factors for mood response to ATD that included recurrent depressive episodes, prior anti-depressant treatment with serotonin-modulating medications, and female gender (Booij et al., 2002). Differences in mood effects in these groups are likely attributable to increased vulnerability to serotonergic fluctuations in populations with certain psychiatric conditions and in females (Booij et al., 2002; Jans et al., 2007; Ruhe et al., 2007). Given that ATD does not appear to affect mood in healthy volunteers without family histories of depression, any effects of ATD on cognition that might be discovered in this population are likely independent of mood changes.

The main aim of this review is to examine the exact impact that ATD, and thus indirectly 5-HT, has on several types of cognition, namely psychomotor processing, declarative memory (episodic and semantic), working memory, executive functions, and attention. Many research groups have used ATD to investigate serotonin's role in various cognitive domains in humans and in some instances, studies have produced conflicting results. Several factors complicate studies on 5-HT and cognition including: (i) variability in sample populations, e.g., healthy volunteers versus depressed patients; (ii) differences in methodology and cognitive assessments; and (iii) the interdependent nature of cognitive operations. Cognitive functions rely on more than one domain and thus no single neuropsychological assessment can tap a single cognitive domain. We will first summarize the literature examined and then we will attempt to dissociate the effects of ATD on memory, executive functions and attention.

\subsection{Previous reviews on ATD and cognitive function}

Several reviews have analyzed the effects of ATD on cognition. Three descriptive reviews summarized the existing research at the time on ATD and cognition (Riedel, 2004; Riedel et al., 2002, 2003). Recently, two articles reviewed the literature on ATD studies involving neuroimaging and electrophysiological techniques (Evers et al., 2007; Fusar-Poli et al., 2006). To our knowledge, this is the first systematic review of ATD effects on cognition.

\section{Serotonin anatomy}

The human 5-HT system consists of an anatomically distinct group of neurons predominantly located in the brainstem raphe nuclei and within parts of the reticular formation (Tork, 1990). The raphe nuclei extend from the medulla oblongata to the midbrain (Tork, 1990). The raphe nuclei in the caudal brainstem project descending pathways to the spinal cord. The median and dorsal raphe nuclei located in the rostral pons and midbrain are the origins of the majority of forebrain serotonergic terminals (Azmitia, 1978). These ascending 5-HT projections innervate the midbrain substantia nigra, basal ganglia and cerebellum, regions involved in motor functions, the limbic system and amygdala, implicated in emotion, the hypothalamus, an autonomic regulatory region involved in the hypothalamicpituitary-adrenal (HPA) axis stress response, the hippocampus, associated with memory operations, and virtually the entire cerebral cortex (Nieuwenhuys, 1985; Stahl, 2008; Tork, 1990). The diffuse nature of the serotonergic system supports the notion that serotonin exerts a tonic modulatory influence on widespread areas of the brain with specificity achieved through interactions with the various subtypes of serotonin receptors (Barnes and Sharp, 1999; Buhot, 1997; Jacobs and Azmitia, 1992). Given the anatomical and functional segregation of the serotonergic pathways, depletion of central 5-HT may have different effects on the various functions and operations mediated by the innervated regions. 


\section{Methods}

\subsection{Selection procedures}

We searched Medline from 1966 to September 2008, and EMBASE and PsycINFO from 1980 to September 2008, using the search terms: "tryptophan depletion" and "cognition", "memory", "attention" or "executive function". The bibliographies of the articles identified were hand-searched for additional articles that met the following criteria. In order to be included in the review, the studies must: (1) be original papers written in English appearing in a peer-reviewed journal, (2) include a comparison condition (ATD versus balanced drink or ATD versus TRP loading) (3) specify inclusion criteria or sample characteristics for the participants (common criteria included co-morbidities, medication status, history of psychiatric illnesses) (4) include cognitive assessments (5) achieve successful TRP depletion during cognitive testing verified with plasma TRP measurements. All articles reporting data on ATD and cognition meeting the above criteria were included.

\subsection{Methodological remarks}

Sixty-six studies met the inclusion criteria. Forty-five included only healthy volunteers, 14 assessed healthy volunteers and psychiatric populations or participants with family histories of mental illness, and seven included solely psychiatric groups. The composition of the TRP free drink varied from containing as little as $25 \mathrm{~g}$ (Booij et al., 2005; Merens et al., 2008) to as much as $104.5 \mathrm{~g}$ (Talbot et al., 2006). Fifty-three articles included assessments of mood and 13 did not include mood assessments. The most commonly used mood assessments were the Profile of Mood States, Visual Analogue Scales, and modifications of the Hamilton Depression Scale.

Mood was mainly affected in the studies that included psychiatric patients or participants with family histories for mental illnesses. In healthy participants without family histories of psychiatric illnesses, only two studies revealed that ATD negatively affected mood (Klaassen et al., 1999; Luciana et al., 2001). In one of these studies, mood response predicted task performance (Klaassen et al., 1999) and in the other mood effects were not systematically related to cognitive outcomes (Luciana et al., 2001). Thus, ATD did not appear to affect mood in healthy volunteers without family histories of depression.

The majority of the studies used repeated-measures crossover designs but in 11 studies, treatment and placebo were administered between-subjects usually to avoid practice or learning effects (Anderson et al., 2003; Harmer et al., 2003; Hayward et al., 2005; LeMarquand et al., 1999; Marsh et al., 2006; Munafo et al., 2006; Rogers et al., 1999a,b, 2003; Rubinsztein et al., 2001; Talbot et al., 2006).

All studies showed that plasma TRP was significantly decreased after ATD as compared to the placebo (Table 1). Plasma

Table 1

Plasma changes after acute tryptophan depletion (ATD) examined in studies included in the systematic review.

\begin{tabular}{|c|c|c|c|c|c|}
\hline \multirow[t]{2}{*}{ Study } & \multirow{2}{*}{$\begin{array}{l}\text { Type of drink, amount of TRP } \\
\text { in balanced condition }\end{array}$} & \multicolumn{2}{|c|}{ Changes in free TRP levels following } & \multicolumn{2}{|c|}{ Changes in TRP $/ \Sigma$ LNAA ratio following } \\
\hline & & ATD & Balanced drink & ATD & Balanced drink \\
\hline Park et al. (1994) & $52 \mathrm{~g}$ AA mixture, $1.15 \mathrm{~g}$ TRP & $\downarrow 79 \%$ a & $\uparrow 7 \%^{\mathrm{a}}$ & Not assessed & Not assessed \\
\hline LeMarquand et al. (1998) & $100 \mathrm{~g}$ AA mixture, $2.3 \mathrm{~g}$ TRP & $\downarrow 81 \%$ & $\uparrow 96 \%$ & Not assessed & Not assessed \\
\hline Klaassen et al. (1999) & $100 \mathrm{~g}$ AA mixture, $3.0 \mathrm{~g}$ TRP & $\downarrow 78 \%^{\mathrm{a}}$ & $\uparrow 12 \%^{\mathrm{a}}$ & $\downarrow 79 \%$ & $\uparrow 2 \%$ \\
\hline LeMarquand et al. (1999) & $100 \mathrm{~g}$ AA mixture, $2.3 \mathrm{~g} \mathrm{TRP}^{\mathrm{b}}$ & $\downarrow 86 \%$ & $\uparrow 57 \%$ & Not assessed & Not assessed \\
\hline $\begin{array}{l}\text { Morris et al. (1999) and } \\
\text { Smith et al. (1999) }\end{array}$ & $100 \mathrm{~g}$ AA mixture, $2.3 \mathrm{~g}$ TRP & $\downarrow 87 \%$ & $\uparrow 28 \%$ & Not assessed & Not assessed \\
\hline Riedel et al. (1999) & $100 \mathrm{~g}$ AA mixture, $3.0 \mathrm{~g}$ TRP & $\downarrow 67 \%^{\mathrm{a}}$ & $\uparrow 21 \%^{\mathrm{a}}$ & $\downarrow 78 \%$ & $\downarrow 17 \%$ \\
\hline $\begin{array}{l}\text { Rogers et al. (1999a) } \\
\text { and Rogers et al. (1999b) }\end{array}$ & $100 \mathrm{~g}$ AA mixture, $2.3 \mathrm{~g} \mathrm{TRP}^{\mathrm{b}}$ & $\downarrow 90 \%$ & Not reported & Not assessed & Not assessed \\
\hline $\begin{array}{l}\text { Porter et al. (2000) } \\
\text { and Porter et al. }(2003 \mathrm{~b})^{\mathrm{f}}\end{array}$ & $52 \mathrm{~g}$ AA mixture, $1.15 \mathrm{~g}$ TRP & $\downarrow 71 \%$ & Not reported & Not assessed & Not assessed \\
\hline Schmitt et al. (2000) & $100 \mathrm{~g}$ AA mixture, $4.6 \mathrm{~g}$ TRP & $\downarrow 63 \%$ & $\uparrow 80 \%$ & $\downarrow 21 \%$ & $\uparrow 20 \%$ \\
\hline Shansis et al. (2000) & $100 \mathrm{~g}$ AA mixture, $2.3 \mathrm{~g}$ & $\downarrow 78 \%^{\mathrm{a}}$ & $\uparrow 25 \%^{\mathrm{a}}$ & Not assessed & Not assessed \\
\hline Golightly et al. (2001) & $100 \mathrm{~g}$ AA mixture, $2.3 \mathrm{~g}$ TRP & $\downarrow 79 \%$ & $\uparrow 248 \%$ & Not reported & Not reported \\
\hline \multirow[t]{2}{*}{$\begin{array}{l}\text { Luciana et al. (2001) } \\
\text { and Burgund et al. }(2003)^{\mathrm{f}}\end{array}$} & $100 \mathrm{~g}$ AA mixture, $10.3 \mathrm{~g} \mathrm{TRP}^{\mathrm{c}}$ & $\downarrow 85 \%$ & Not applicable & Not assessed & Not applicable \\
\hline & & $\uparrow 936 \%$ a,c & & & \\
\hline Rubinsztein et al. (2001) & $53 \mathrm{~g}$ AA mixture, $2.0 \mathrm{~g} \mathrm{TRP}^{\mathrm{b}}$ & $\downarrow 81 \%^{\mathrm{a}}$ & $\uparrow 32 \%^{\mathrm{a}}$ & Not assessed & Not assessed \\
\hline Crean et al. (2002) & $100 \mathrm{~g}$ AA mixture, $2.3 \mathrm{~g}$ TRP & $\downarrow 87 \%$ & $\uparrow 98 \%$ & Not assessed & Not assessed \\
\hline $\begin{array}{l}\text { Harrison et al. (2002) } \\
\text { and Harrison et al. }(2004)^{\mathrm{f}}\end{array}$ & $86 \mathrm{~g}$ AA mixture, $1.92 \mathrm{~g}$ TRP & $\downarrow 97 \%$ & $\uparrow 74 \%$ & Not assessed & Not assessed \\
\hline Hughes et al. (2002) & $100 \mathrm{~g}$ AA mixture, $2.3 \mathrm{~g}$ TRP & $\downarrow 80 \%$ & "No difference" & Not assessed & Not assessed \\
\hline Klaassen et al. (2002) & $100 \mathrm{~g}$ AA mixture, $3.0 \mathrm{~g}$ TRP & $\downarrow 67 \%^{\mathrm{a}}$ & Not reported & $\downarrow 78 \%$ & Not reported \\
\hline McAllister-Williams et al. (2002) & $100 \mathrm{~g}$ AA mixture, $2.3 \mathrm{~g}$ TRP & $\downarrow 84 \%$ & $\uparrow 71 \%$ & Not assessed & Not assessed \\
\hline Murphy et al. (2002) & $86 \mathrm{~g}$ AA mixture, $1.9 \mathrm{~g}$ TRP & $\downarrow 75 \%$ & "No changes" & Not assessed & Not assessed \\
\hline Sobczak et al. (2002) & $75 \mathrm{~g}$ AA mixture, $3.0 \mathrm{~g}$ TRP & $\downarrow 57 \% \mathrm{a}^{\mathrm{a}}$ & $\uparrow 36 \%^{\mathrm{a}}$ & $\downarrow 70 \%$ & $\uparrow 10 \%$ \\
\hline Stewart et al. (2002) & $102 \mathrm{~g}$ AA mixture, $2.3 \mathrm{~g} \mathrm{TRP}^{\mathrm{d}}$ & $\downarrow 83 \%$ & $\uparrow 156 \%$ & Not assessed & Not assessed \\
\hline $\begin{array}{l}\text { Walderhaug et al. (2002) } \\
\text { and Walderhaug et al. (2008) }\end{array}$ & $100 \mathrm{~g}$ AA mixture, $2.3 \mathrm{~g}$ TRP & $\downarrow 85 \%$ & $\uparrow 151 \%$ & $\downarrow 92 \%$ & $\uparrow 24 \%$ \\
\hline Anderson et al. (2003) & $100 \mathrm{~g}$ AA mixture, $2.3 \mathrm{~g}$ TRP ${ }^{\mathrm{b}, \mathrm{d}}$ & $\downarrow 86 \%$ & $\uparrow 128 \%$ & Not assessed & Not assessed \\
\hline Gallagher et al. (2003) & $100 \mathrm{~g}$ AA mixture, $2.3 \mathrm{~g}$ TRP & $\downarrow 84 \%$ & $\uparrow 71 \%$ & Not assessed & Not assessed \\
\hline Harmer et al. (2003) & $100 \mathrm{~g}$ AA mixture, $2.3 \mathrm{~g}$ TRP ${ }^{\mathrm{b}, \mathrm{d}}$ & $\downarrow 83 \%^{\mathrm{a}}$ & Not reported & Not assessed & Not assessed \\
\hline Hughes et al. (2003) & $52 \mathrm{~g}$ AA mixture, $1.15 \mathrm{~g}$ TRP & $\downarrow 64 \%$ & $\uparrow 72 \%$ & Not assessed & Not assessed \\
\hline Rogers et al. (2003) & $100 \mathrm{~g}$ AA mixture, $2.3 \mathrm{~g} \mathrm{TRP}{ }^{\mathrm{b}, \mathrm{d}}$ & $\downarrow 83 \%^{\mathrm{a}}$ & $\uparrow 78 \%^{\mathrm{a}}$ & Not assessed & Not assessed \\
\hline Kilkens et al. (2004) & $75 \mathrm{~g}$ AA mixture, $3.0 \mathrm{~g}$ TRP & $\downarrow 63 \%^{\mathrm{a}}$ & $\uparrow 49 \%^{\mathrm{a}}$ & $\downarrow 81 \%$ & $\downarrow 13 \%$ \\
\hline \multirow{2}{*}{ Booij et al. (2005) } & $100 \mathrm{~g}$ AA mixture, $2.3 \mathrm{~g}$ TRP & $\downarrow 86 \%^{\mathrm{a}}$ & Not applicable & $\downarrow 93 \%$ & Not applicable \\
\hline & $25 \mathrm{~g}$ AA mixture, $0.58 \mathrm{~g}$ TRP & $\downarrow 47 \%^{\mathrm{a}}$ & & $\downarrow 42 \%$ & \\
\hline Clark et al. (2005) & $75 \mathrm{~g}$ AA mixture, $3.0 \mathrm{~g} \mathrm{TRP}^{\mathrm{d}}$ & $\downarrow 61 \%^{\mathrm{a}}$ & $\uparrow 68 \%^{\mathrm{a}}$ & $68 \%$ & $\uparrow 13 \%$ \\
\hline Cools et al. (2005a) & $75 \mathrm{~g}$ AA mixture, $3.0 \mathrm{~g} \mathrm{TRP}^{\mathrm{e}}$ & Not reported & Not reported & $\downarrow 73 \%$ & $\downarrow 21 \%$ \\
\hline Cools et al. (2005b) & $75 \mathrm{~g}$ AA mixture, $3.0 \mathrm{~g}$ TRP & $\downarrow 64 \%^{\mathrm{a}}$ & $\uparrow 44 \%^{\mathrm{a}}$ & $75 \%$ & $\downarrow 11 \%$ \\
\hline Evers et al. (2005a) & $75 \mathrm{~g}$ AA mixture, $3.0 \mathrm{~g}$ TRP & $\downarrow 64 \%$ & $\uparrow 50 \%$ & $\downarrow 74 \%$ & $\downarrow 16 \%$ \\
\hline
\end{tabular}


Table 1 (Continued)

\begin{tabular}{|c|c|c|c|c|c|}
\hline \multirow[t]{2}{*}{ Study } & \multirow{2}{*}{$\begin{array}{l}\text { Type of drink, amount of TRP } \\
\text { in balanced condition }\end{array}$} & \multicolumn{2}{|c|}{ Changes in free TRP levels following } & \multicolumn{2}{|c|}{ Changes in TRP/ $\Sigma$ LNAA ratio following } \\
\hline & & ATD & Balanced drink & ATD & Balanced drink \\
\hline Evers et al. (2005b) & $\begin{array}{l}100 \mathrm{~g} \text { gelatin-based mixture, } \\
1.2 \mathrm{~g} \text { TRP }\end{array}$ & $\downarrow 74 \% \mathrm{a}$ & $\uparrow 8 \% \mathrm{a}$ & $\downarrow 82 \%$ & $\downarrow 2 \%$ \\
\hline Hayward et al. (2005) & $31 \mathrm{~g} \mathrm{AA}$ mixture, $2.0 \mathrm{~g} \mathrm{TRP}^{\mathrm{b}}$ & $\downarrow 65 \%^{\mathrm{a}}$ & $\uparrow 48 \%^{\mathrm{a}}$ & $\downarrow 87 \%$ & $\downarrow 23 \%$ \\
\hline Horacek et al. (2005) & $103 \mathrm{~g}$ AA mixture, $2.3 \mathrm{~g}$ TRP & $\downarrow 89 \%^{\mathrm{a}}$ & Not reported & Not assessed & Not assessed \\
\hline Porter et al. (2005) & $100 \mathrm{~g}$ AA mixture, $2.3 \mathrm{~g}$ TRP $^{\mathrm{d}}$ & $\downarrow 73 \%$ & $\uparrow 277 \%$ & Not assessed & Not assessed \\
\hline Rubia et al. (2005) & $100 \mathrm{~g}$ AA mixture, $2.3 \mathrm{~g}$ TRP & $\downarrow 80 \%^{\mathrm{a}}$ & $\uparrow 63 \%^{\mathrm{a}}$ & Not assessed & Not assessed \\
\hline Allen et al. (2006) & $100 \mathrm{~g}$ AA mixture, $2.3 \mathrm{~g}$ TRP & $\downarrow 79 \%^{\mathrm{a}}$ & $\uparrow 71 \%^{\mathrm{a}}$ & Not assessed & Not assessed \\
\hline Amin et al. (2006) & $\begin{array}{l}\text { AA mixture, composition not } \\
\text { specified }\end{array}$ & $\downarrow 84 \%$ & $\uparrow 123 \%$ & Not assessed & Not assessed \\
\hline Evers et al. (2006a) & 75 g AA mixture, $3.0 \mathrm{~g}$ TRP & $\downarrow 80 \%^{\mathrm{a}}$ & $\uparrow 202 \%$ a & $\downarrow 91 \%$ & $\uparrow 17 \%$ \\
\hline Evers et al. (2006b) & $75 \mathrm{~g}$ AA mixture, $3.0 \mathrm{~g}$ TRP & $\downarrow 59 \%^{\mathrm{a}}$ & $\uparrow 128 \%{ }^{\mathrm{a}}$ & $77 \%$ & $\uparrow 15 \%$ \\
\hline Marsh et al. (2006) & $\begin{array}{l}31.5 \mathrm{~g} \text { AA in capsules, } \\
31 \text { g lactose (balanced drink) }\end{array}$ & $\downarrow 80 \%$ & $\downarrow 38 \%$ & Not reported & Not reported \\
\hline Munafo et al. (2006) & $31 \mathrm{~g} \mathrm{AA}$ mixture, $2.0 \mathrm{~g} \mathrm{TRP}^{\mathrm{b}}$ & Not reported & Not reported & Not assessed & Not assessed \\
\hline Roiser et al. (2006) & $75 \mathrm{~g}$ AA mixture, $3.0 \mathrm{~g}$ TRP ${ }^{\mathrm{d}}$ & $\downarrow 69 \%^{\mathrm{a}}$ & $\uparrow 65 \%{ }^{\mathrm{a}}$ & $\downarrow 63 \%$ & $\uparrow 36 \%$ \\
\hline Scholtissen et al. (2006) & $75 \mathrm{~g}$ AA mixture, $3.0 \mathrm{~g}$ TRP & $\downarrow 58 \%$ & Not reported & $\downarrow 75 \%$ & $\uparrow 39 \%$ \\
\hline Talbot et al. (2006) & $105 \mathrm{~g}$ AA mixture, $2.2 \mathrm{~g} \mathrm{TRP}^{\mathrm{b}, \mathrm{d}}$ & $\downarrow 71 \%$ & $\uparrow 66 \%$ & Not assessed & Not assessed \\
\hline van der Veen et al. (2006) & $75 \mathrm{~g}$ AA mixture, $3.0 \mathrm{~g}$ TRP & $\downarrow 62 \%^{\mathrm{a}}$ & $\uparrow 131 \%^{\mathrm{a}}$ & $\downarrow 80 \%$ & $\uparrow 10 \%$ \\
\hline Dougherty et al. (2007) & $50 \mathrm{~g}$ AA mixture, $5.15 \mathrm{~g} \mathrm{TRP}^{\mathrm{c}}$ & $\begin{array}{l}\downarrow 76 \%^{\mathrm{a}} \\
\uparrow 237 \%^{\mathrm{a}, \mathrm{c}}\end{array}$ & Not applicable & $\begin{array}{l}\downarrow 83 \% \\
\uparrow 127 \% \mathrm{c}\end{array}$ & Not applicable \\
\hline Epperson et al. (2007) & ? AA mixture, $2.3 \mathrm{~g}$ TRP & $88 \%$ & $\uparrow 173 \%$ & Not assessed & Not assessed \\
\hline Fusar-Poli et al. (2007) & $100 \mathrm{~g}$ AA mixture, $2.3 \mathrm{~g}$ TRP & $\downarrow 79 \%^{\mathrm{a}}$ & Not reported & Not assessed & Not assessed \\
\hline Hitsman et al. (2007) & 103 g AA mixture,? TRP & $\downarrow 80 \%$ & $\downarrow 19 \%$ & $\downarrow 90 \%$ & $\uparrow 10 \%$ \\
\hline Kulz et al. (2007) & $75 \mathrm{~g}$ AA mixture, $3.0 \mathrm{~g}$ TRP & $\downarrow 46 \%$ & $\uparrow 51 \%$ & Not assessed & Not assessed \\
\hline Roiser et al. (2007) & $75 \mathrm{~g}$ AA mixture, $3.0 \mathrm{~g}$ TRP & $\downarrow 62 \%^{\mathrm{a}}$ & $\uparrow 75 \%^{\mathrm{a}}$ & $\downarrow 64 \%$ & $\uparrow 33 \%$ \\
\hline Scholes et al. (2007) & $100 \mathrm{~g}$ AA mixture, $2.3 \mathrm{~g}$ TRP & $\downarrow 84 \%$ & $\uparrow 142 \%$ & $\downarrow 93 \%$ & $\downarrow 20 \%$ \\
\hline van der Veen et al. (2007) & $75 \mathrm{~g}$ AA mixture, $3.0 \mathrm{~g}$ TRP & $76 \%^{\mathrm{a}}$ & $\uparrow 222 \%$ & $\downarrow 92 \%$ & $\uparrow 20 \%$ \\
\hline Williams et al. (2007) & $100 \mathrm{~g}$ AA mixture, $2.3 \mathrm{~g}$ TRP & Not reported & Not reported & Not assessed & Not assessed \\
\hline Merens et al. (2008) & $\begin{array}{l}103 \mathrm{~g} \text { AA mixture, } 2.3 \mathrm{~g} \text { TRP } \\
26 \mathrm{~g} \text { AA mixture, } 0.58 \mathrm{~g} \text { TRP }\end{array}$ & $\begin{array}{l}\downarrow 84 \%^{\mathrm{a}} \\
\downarrow 60 \%^{\mathrm{a}}\end{array}$ & Not applicable & $\begin{array}{l}\downarrow 91 \% \\
\downarrow 59 \%\end{array}$ & Not applicable \\
\hline Roiser et al. (2008) & $32 \mathrm{~g} \mathrm{AA}$ in capsules, $1.2 \mathrm{~g}$ TRP & $\downarrow 67 \%^{\mathrm{a}}$ & $\uparrow 85 \%^{\mathrm{a}}$ & $87 \%$ & $\downarrow 37 \%$ \\
\hline Sambeth et al. (2009) & $\begin{array}{l}100 \mathrm{~g} \text { gelatin-based mixture, } \\
1.21 \mathrm{~g} \text { TRP }\end{array}$ & $\downarrow 60 \%$ & $\uparrow 36 \%$ & $\downarrow 63 \%$ & $\uparrow 24 \%$ \\
\hline
\end{tabular}

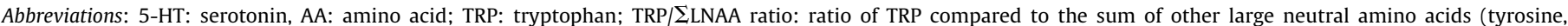
phenylalanine, leucine, isoleuince, and valine).

a Value for total plasma tryptophan.

b Treatment administered between-subjects.

c Tryptophan loading.

d $80 \%$ of mixture for female participants.

e Mixed parallel group and crossover design.

f Studies that present results from the same group of participants.

TRP levels were reported as either changes in total plasma TRP, free plasma TRP or in the ratio of plasma TRP to large neutral amino acids. Three studies intentionally included a TRP loading condition (Burgund et al., 2003; Dougherty et al., 2007; Luciana et al., 2001). The reduction in plasma TRP levels following ATD varied from as low as $46 \%$ (Kulz et al., 2007) to as high as $97 \%$ (Harrison et al., 2002, 2004). In several studies, plasma TRP concentration increased by over $100 \%$ following the balanced drink (placebo). However, of these studies that also reported to the ratio of plasma TRP to large neutral amino acids, the highest increase in the ratio was $24 \%$ (Walderhaug et al., 2002, 2008). Given that the plasma TRP to large neutral amino acid ratio is believed to be a more accurate measure of TRP levels in the brain (Fernstrom, 1979), these studies that found large increases in plasma TRP following the balanced drink cannot be definitively considered to be TRP loading.

Table 2

Acute tryptophan depletion studies including tasks assessing psychomotor processing.

\begin{tabular}{|c|c|c|c|c|}
\hline Study & Participants/design & $\begin{array}{l}\text { Gender F:M } \\
\text { (mean age) }\end{array}$ & Tasks & Effects of ATD \\
\hline \multicolumn{5}{|l|}{ Perceptual processing } \\
\hline Stewart et al. (2002) & $\begin{array}{l}32 \text { healthy volunteers } \\
\text { Neuroticism: } \\
17 \text { high, } 15 \text { low } \\
\text { Crossover design }\end{array}$ & GNS (22) & Visual Change Detection Task & No effect \\
\hline Riedel et al. (1999) & $\begin{array}{l}27 \text { healthy volunteers } \\
\text { Depression: } \\
16 \text { FHP, } 11 \text { FHN } \\
\text { Crossover design }\end{array}$ & $15 \mathrm{~F}: 12 \mathrm{M}(31)$ & Visual Search Task & No effect on perceptual sensitivity or RT \\
\hline Walderhaug et al. (2002) & $\begin{array}{l}24 \text { healthy volunteers } \\
\text { Crossover design }\end{array}$ & $24 \mathrm{M}(25)$ & $\begin{array}{l}\text { Continuous Performance } \\
\text { Task - identical pairs }\end{array}$ & $\begin{array}{l}\text { Lowered response criterion (disinhibition) } \\
\text { during number mode } \\
\text { Fewer hits and lower perceptual sensitivity } \\
\text { during shape mode }\end{array}$ \\
\hline
\end{tabular}


Table 2 (Continued)

\begin{tabular}{|c|c|c|c|c|}
\hline Study & Participants/design & $\begin{array}{l}\text { Gender F:M } \\
\text { (mean age) }\end{array}$ & Tasks & Effects of ATD \\
\hline Walderhaug et al. (2008) & Crossover design & $24 \mathrm{M}(25)$ & $\begin{array}{l}\text { Continuous Performance } \\
\text { Task - identical pairs }\end{array}$ & $\begin{array}{l}\text { When task was novel, increased false alarms } \\
\text { in number and shape modes, lowered } \\
\text { response criterion in number mode, and } \\
\text { decreased perceptual sensitivity in shape mode }\end{array}$ \\
\hline Harrison et al. (2002) & $\begin{array}{l}13 \text { healthy volunteers } \\
\text { Crossover design }\end{array}$ & $13 \mathrm{~F}(22)$ & $\begin{array}{l}\text { Inspection time with } \\
\text { backward masking }\end{array}$ & $\begin{array}{l}\text { No effect on inspection times or total trials } \\
\text { needed to reach accuracy threshold }\end{array}$ \\
\hline Harrison et al. (2004) & $\begin{array}{l}13 \text { healthy volunteers } \\
\text { Crossover design }\end{array}$ & $13 \mathrm{~F}(22)$ & Critical flicker fusion & $\begin{array}{l}\text { Lower (improved) performance thresholds } \\
\text { for flicker fusion discrimination }\end{array}$ \\
\hline Park et al. (1994) & $\begin{array}{l}12 \text { healthy volunteers } \\
\text { Crossover design }\end{array}$ & $12 \mathrm{M}(29)$ & $\begin{array}{l}\text { Rapid visual information } \\
\text { processing }\end{array}$ & $\begin{array}{l}\text { No main effect on correct signal detection } \\
\text { or response bias } \\
\text { Decreased response latencies when task } \\
\text { was novel and increased response latencies } \\
\text { when task was familiar }\end{array}$ \\
\hline $\begin{array}{l}\text { Psychomotor processing } \\
\text { Sobczak et al. (2002) }\end{array}$ & $\begin{array}{l}45 \text { healthy volunteers } \\
\text { Bipolar disorder: } \\
30 \text { FHP, } 15 \text { FHN } \\
\text { Crossover design }\end{array}$ & $\begin{array}{l}22 \mathrm{~F}: 8 \mathrm{M} \\
11 \mathrm{~F}: 4 \mathrm{M} \\
(41)\end{array}$ & Concept-Shifting Task (CST) & No effect \\
\hline Stewart et al. (2002) & $\begin{array}{l}32 \text { healthy volunteers } \\
\text { Neuroticism: } \\
17 \text { high, } 15 \text { low } \\
\text { Crossover design }\end{array}$ & GNS (22) & Digit-Symbol Substitution Test & $\begin{array}{l}\text { Improved performance in both and high } \\
\text { and low neuroticism groups }\end{array}$ \\
\hline Scholtissen et al. (2006) & $\begin{array}{l}15 \text { healthy elderly } \\
15 \text { Parkinson's patients } \\
\text { Crossover design }\end{array}$ & $\begin{array}{l}6 \mathrm{~F}: 9 \mathrm{M} \\
6 \mathrm{~F}: 9 \mathrm{M} \\
(61)\end{array}$ & $\begin{array}{l}\text { Simple RT } \\
\text { Finger Precuing Task } \\
\text { Concept-Shifting Task (CST) }\end{array}$ & $\begin{array}{l}\text { Decreased RT in healthy elderly and } \\
\text { Parkinson's patients } \\
\text { Decreased RT in healthy elderly and } \\
\text { Parkinson's patients } \\
\text { No effect on CST A or B (motor scores) }\end{array}$ \\
\hline Riedel et al. (1999) & $\begin{array}{l}27 \text { healthy volunteers } \\
\text { Depression: } \\
16 \text { FHP, } 11 \text { FHN } \\
\text { Crossover design }\end{array}$ & $15 \mathrm{~F}: 12 \mathrm{M}(31)$ & Motor choice RT & No effect \\
\hline Hughes et al. (2003) & $\begin{array}{l}20 \text { healthy volunteers } \\
\text { Crossover design }\end{array}$ & $20 \mathrm{M}(24)$ & Trail Making Test (TMT) & No effect on TMT A latencies (motor scores) \\
\hline Booij et al. (2005) & $\begin{array}{l}20 \text { remitted depressed } \\
\text { patients } \\
\text { Crossover design }\end{array}$ & $9 F: 11 \mathrm{M}(49)$ & Left/right choice RT & No effect \\
\hline Schmitt et al. (2000) & $\begin{array}{l}17 \text { healthy volunteers } \\
\text { Crossover design }\end{array}$ & $9 \mathrm{~F}: 8 \mathrm{M}(23)$ & $\begin{array}{l}\text { Symbol Digit Substitution Test } \\
\text { Motor Choice RT }\end{array}$ & $\begin{array}{l}\text { No effect } \\
\text { No effect }\end{array}$ \\
\hline Gallagher et al. (2003) & Crossover design & $15 \mathrm{M}(22)$ & Trail Making Test (TMT) & $\begin{array}{l}\text { Decreased response times in TMT A } \\
\text { (further decreased when ATD given on } \\
\text { second day) }\end{array}$ \\
\hline Harrison et al. (2004) & $\begin{array}{l}13 \text { healthy volunteers } \\
\text { Crossover design }\end{array}$ & $13 \mathrm{~F}(22)$ & $\begin{array}{l}\text { Simple RT } \\
\text { Choice RT }\end{array}$ & $\begin{array}{l}\text { No effect } \\
\text { No effect }\end{array}$ \\
\hline Kulz et al. (2007) & $\begin{array}{l}7 \text { OCD patients } \\
\text { Crossover design }\end{array}$ & $4 \mathrm{~F}: 3 \mathrm{M}(28)$ & Trail Making Test (TMT) & No effect on TMT A latencies \\
\hline Motor abilities & & & & \\
\hline Porter et al. (2003a,b) & $\begin{array}{l}16 \text { healthy elderly } \\
16 \text { Alzheimer's patients } \\
\text { Crossover design }\end{array}$ & $\begin{array}{l}8 \mathrm{~F}: 8 \mathrm{M} \\
10 \mathrm{~F}: 6 \mathrm{M} \\
(74)\end{array}$ & Motor Screening Task & No effect on median latency \\
\hline Luciana et al. (2001) & $\begin{array}{l}19 \text { healthy volunteers } \\
\text { Crossover design }\end{array}$ & GNS (22) & $\begin{array}{l}\text { Finger tapping } \\
\text { Grooved pegboard }\end{array}$ & $\begin{array}{l}\text { No effect of ATD or tryptophan loading } \\
\text { during finger tapping } \\
\text { ATD sped completion times during groove } \\
\text { pegboard compared to tryptophan loading. } \\
\text { Tryptophan loading led to more pegs } \\
\text { dropped compared to ATD }\end{array}$ \\
\hline
\end{tabular}

Abbreviations: ATD: acute tryptophan depletion; FHP: family history positive; FHN: family history negative; GNS: genders not specified; RT: reaction times. 
Table 3

Acute tryptophan depletion studies including tasks assessing verbal learning.

\begin{tabular}{|c|c|c|c|c|c|c|}
\hline \multirow[t]{2}{*}{ Study } & \multirow[t]{2}{*}{ Participants/design } & \multirow{2}{*}{$\begin{array}{l}\text { Gender F:M } \\
\text { (mean age) }\end{array}$} & \multirow[t]{2}{*}{ Task parameters } & \multicolumn{3}{|l|}{ Effects of ATD } \\
\hline & & & & Immediate recall & Delayed recall & Delayed recognition \\
\hline $\begin{array}{l}\text { Visual verbal learning task } \\
\text { Sobczak et al. (2002) }\end{array}$ & $\begin{array}{l}45 \text { healthy volunteers } \\
\text { Bipolar disorder: } \\
30 \text { FHP, } 15 \text { FHN } \\
\text { Crossover design }\end{array}$ & $\begin{array}{l}22 \mathrm{~F}: 8 \mathrm{M} \\
11 \mathrm{~F}: 4 \mathrm{M} \\
(41)\end{array}$ & $\begin{array}{l}\text { Visual VLT ( } 30 \text { word) } \\
\text { Delay: } 30 \mathrm{~min}, 7 \mathrm{~h}\end{array}$ & No effect & t7-t7: Impaired & No effect \\
\hline Scholtissen et al. (2006) & $\begin{array}{l}15 \text { healthy elderly } \\
15 \text { Parkinson's patients } \\
\text { Crossover design }\end{array}$ & $\begin{array}{l}6 \mathrm{~F}: 9 \mathrm{M} \\
6 \mathrm{~F}: 9 \mathrm{M} \\
(61)\end{array}$ & $\begin{array}{l}\text { Visual VLT ( } 15 \text { word) } \\
\text { Delay: } 25 \mathrm{~min}\end{array}$ & $\begin{array}{l}\text { Trend towards } \\
\text { impairment }\end{array}$ & Impaired & Impaired \\
\hline Riedel et al. (1999) & $\begin{array}{l}27 \text { healthy volunteers } \\
\text { Depression: } \\
16 \text { FHP, } 11 \text { FHN } \\
\text { Crossover design }\end{array}$ & $15 \mathrm{~F}: 12 \mathrm{M}(31)$ & $\begin{array}{l}\text { Visual VLT ( } 30 \text { word) } \\
\text { Delay: } 30 \mathrm{~min}, 18 \mathrm{~h}\end{array}$ & No effect & $\begin{array}{l}\text { t6-t6: Impaired } \\
\text { t6-t24: Impaired }\end{array}$ & $\begin{array}{l}\text { t6-t6: Intact } \\
\text { t6-t24: Impaired } \\
\text { Slower RT }\end{array}$ \\
\hline Schmitt et al. (2000) & $\begin{array}{l}17 \text { healthy volunteers } \\
\text { Crossover design }\end{array}$ & $9 F: 8 M(23)$ & $\begin{array}{l}\text { Visual VLT ( } 30 \text { word) } \\
\text { Delay: } 30 \mathrm{~min}, 4 \mathrm{~h}\end{array}$ & No effect & $\begin{array}{l}\text { t5-t5: Impaired } \\
\text { t5-t9: Impaired }\end{array}$ & $\begin{array}{l}\text { t5-t5: Impaired } \\
\text { t5-t9: Impaired }\end{array}$ \\
\hline Evers et al. (2005b) & $\begin{array}{l}15 \text { healthy volunteers } \\
\text { Crossover design }\end{array}$ & $12 \mathrm{~F}: 3 \mathrm{M}(22)$ & $\begin{array}{l}\text { Visual VLT ( } 30 \text { word) } \\
\text { Delayed: } 30 \mathrm{~min}\end{array}$ & No effect & No effect & $\begin{array}{l}\text { No effect on accuracy } \\
\text { Increased RT }\end{array}$ \\
\hline Klaassen et al. (1999) & $\begin{array}{l}13 \text { healthy volunteers } \\
\text { Crossover design }\end{array}$ & $10 F: 3 M(27)$ & $\begin{array}{l}\text { Visual VLT ( } 30 \text { word) } \\
\text { Delay: } 30 \mathrm{~min}, 18 \mathrm{~h}\end{array}$ & No effect & $\begin{array}{l}\text { t6-t6: Intact } \\
\text { t6-t24: Impaired }\end{array}$ & $\begin{array}{l}\text { t6-t6: No effect } \\
\text { t6-t24: No effect }\end{array}$ \\
\hline Harrison et al. (2004) & $\begin{array}{l}13 \text { healthy volunteers } \\
\text { Crossover design }\end{array}$ & $13 F(22)$ & $\begin{array}{l}\text { Visual VLT ( } 15 \text { word) } \\
\text { Delay: } 20 \mathrm{~min}\end{array}$ & No effect & Impaired & No effect \\
\hline Sambeth et al. (2009) & $\begin{array}{l}13 \text { healthy volunteers } \\
\text { Crossover design }\end{array}$ & $8 \mathrm{~F}: 5 \mathrm{M}(22)$ & $\begin{array}{l}\text { Visual VLT ( } 30 \text { word) } \\
\text { Delay: } 30 \mathrm{~min}\end{array}$ & No effect & Impaired & No effect \\
\hline $\begin{array}{l}\text { Auditory verbal learning ta } \\
\text { Porter et al. (2005) }\end{array}$ & $\begin{array}{l}16 \text { recovered depressed } \\
\text { patients } \\
\text { Crossover design }\end{array}$ & $\begin{array}{l}10 \mathrm{~F}: 6 \mathrm{M} \\
(70)\end{array}$ & $\begin{array}{l}\text { (number of words } \\
\text { and delay time } \\
\text { not specified) }\end{array}$ & $\begin{array}{l}\text { Impaired for } \\
\text { some word } \\
\text { lists }\end{array}$ & Impaired & Not tested \\
\hline Hughes et al. (2003) & $\begin{array}{l}20 \text { healthy volunteers } \\
\text { Crossover design }\end{array}$ & $20 \mathrm{M}(24)$ & $\begin{array}{l}\text { Rey's auditory } \\
\text { VLT ( } 15 \text { word) } \\
\text { Delay: } 30 \mathrm{~min}\end{array}$ & No effect & No effect & No effect \\
\hline Merens et al. (2008) & $\begin{array}{l}18 \text { remitted depressed } \\
\text { patients } \\
\text { Crossover design }\end{array}$ & $16 \mathrm{~F}: 2 \mathrm{M}(44)$ & $\begin{array}{l}\text { Auditory VLT } \\
\text { (15 words) } \\
\text { Delay: } 15 \text { min }\end{array}$ & Impaired & Impaired & Not tested \\
\hline Porter et al. (2003a,b) & $\begin{array}{l}16 \text { healthy elderly } \\
\text { Crossover design }\end{array}$ & $\begin{array}{l}8 \mathrm{~F}: 8 \mathrm{M} \\
10 \mathrm{~F}: 6 \mathrm{M} \\
(74)\end{array}$ & $\begin{array}{l}\text { Rey's Auditory } \\
\text { VLT ( } 15 \text { word) } \\
\text { Delay: } 20 \text { min }\end{array}$ & No effect & No effect & No effect \\
\hline Shansis et al. (2000) & $\begin{array}{l}12 \text { healthy volunteers } \\
\text { Mental disorders: } \\
5 \text { FHP, } 7 \text { FHN } \\
\text { Crossover design }\end{array}$ & GNS (24) & $\begin{array}{l}\text { Rey's Auditory } \\
\text { VLT ( } 15 \text { word) } \\
\text { (duration of delay } \\
\text { not specified) }\end{array}$ & No effect & No effect & Not tested \\
\hline Kulz et al. (2007) & Crossover design & $4 \mathrm{~F}: 3 \mathrm{M}(28)$ & $\begin{array}{l}\text { Auditory VLT } \\
\text { (number of words } \\
\text { not specified) } \\
\text { Delay: } 30 \mathrm{~min}\end{array}$ & No effect & Not tested & No effect \\
\hline Hayward et al. (2005) & $\begin{array}{l}24 \text { healthy controls } \\
24 \text { recovered depressed } \\
\text { patients } \\
\text { Parallel group design }\end{array}$ & $\begin{array}{l}\text { 14F:10M } \\
14 \mathrm{~F}: 10 \mathrm{M} \\
(38)\end{array}$ & $\begin{array}{l}\text { Auditory VLT } \\
\text { (15 word) } \\
\text { Delay: } 15 \text { min }\end{array}$ & $\begin{array}{l}\text { No effect on } \\
\text { controls } \\
\text { Impaired in } \\
\text { patients }\end{array}$ & No effect & No effect \\
\hline \multicolumn{7}{|c|}{ Affective visual verbal learning task } \\
\hline Roiser et al. (2007) & $\begin{array}{l}\text { 5-HT genotype: } \\
15 \text { ss, } 15 \text { ll } \\
\text { Crossover design }\end{array}$ & $13 \mathrm{~F}: 17 \mathrm{M}(27)$ & $\begin{array}{l}\text { Affective directed } \\
\text { forgetting } \\
\text { ( } 36 \text { words) } \\
\text { Delay: } 20 \text { min }\end{array}$ & $\begin{array}{l}\text { Impaired } \\
\text { only in ss } \\
\text { genotype }\end{array}$ & Not tested & $\begin{array}{l}\text { No effect on } \\
\text { accuracy or RT }\end{array}$ \\
\hline
\end{tabular}


Table 3 (Continued)

\begin{tabular}{|c|c|c|c|c|c|c|}
\hline \multirow[t]{2}{*}{ Study } & \multirow[t]{2}{*}{ Participants/design } & \multirow{2}{*}{$\begin{array}{l}\text { Gender F:M } \\
\text { (mean age) }\end{array}$} & \multirow[t]{2}{*}{ Task parameters } & \multicolumn{3}{|l|}{ Effects of ATD } \\
\hline & & & & Immediate recall & Delayed recall & Delayed recognition \\
\hline \multirow[t]{3}{*}{ Kilkens et al. (2004) } & 14 healthy controls & $8 \mathrm{~F}: 6 \mathrm{M}$ & $\begin{array}{l}\text { Affective visual } \\
\text { VLT ( } 30 \text { word) }\end{array}$ & Impaired & $\begin{array}{l}\text { Impaired } \\
\text { recall of } \\
\text { positive } \\
\text { words }\end{array}$ & Not tested \\
\hline & $\begin{array}{l}14 \text { irritable bowel } \\
\text { syndrome patients }\end{array}$ & $8 \mathrm{~F}: 6 \mathrm{M}$ & Delay: 30 min & & & \\
\hline & Crossover design & $(34)$ & $\begin{array}{l}12 \text { positive, } 12 \\
\text { negative, } \\
6 \text { neutral words }\end{array}$ & & & \\
\hline \multirow[t]{4}{*}{ Klaassen et al. (2002) } & 27 healthy volunteers & 15F:12M (31) & $\begin{array}{l}\text { Affective Visual } \\
\text { VLT ( } 30 \text { word) }\end{array}$ & No effect & $\begin{array}{l}\text { t6-t6: } \\
\text { Impaired } \\
\text { (neutral words) }\end{array}$ & Not tested \\
\hline & Major affective disorder: & & Delay: 30 min, 18 h & & $\begin{array}{l}\text { t6-t24: } \\
\text { Impaired } \\
\text { (positive words) }\end{array}$ & \\
\hline & 16 FHP, 11 FHN & & $\begin{array}{l}12 \text { positive, } 12 \\
\text { negative, }\end{array}$ & & & \\
\hline & Crossover & & 6 neutral words & & & \\
\hline \multirow[t]{2}{*}{$\begin{array}{l}\text { van der Veen } \\
\text { et al. (2006) }\end{array}$} & 14 healthy volunteers & $14 \mathrm{M}(23)$ & $\begin{array}{l}\text { Affective Visual } \\
\text { VLT ( } 52 \text { word) }\end{array}$ & Not tested & Not tested & $\begin{array}{l}\text { Decreased } \\
\text { recognition of } \\
\text { words rated } \\
\text { positively }\end{array}$ \\
\hline & Crossover design & & $\begin{array}{l}\text { Delay: } 20 \text { min } \\
\text { Participants rate } \\
\text { valence of words } \\
\text { during encoding } \\
\text { (fMRI) }\end{array}$ & & & \\
\hline \multirow[t]{3}{*}{ Hayward et al. (2005) } & 24 healthy controls & 14F:10M & $\begin{array}{l}\text { Emotional Memory } \\
\text { Task }\end{array}$ & No effect & Not tested & Not tested \\
\hline & $\begin{array}{l}24 \text { recovered } \\
\text { depressed patients }\end{array}$ & 14F:10M & Delay: 5 min & & & \\
\hline & Parallel group design & $(38)$ & & & & \\
\hline \multicolumn{7}{|c|}{ Verbal paired associates learning } \\
\hline \multirow[t]{2}{*}{ Hughes et al. (2003) } & 20 healthy volunteers & $20 \mathrm{M}(24)$ & $\begin{array}{l}\text { Verbal paired } \\
\text { associates learning }\end{array}$ & $\begin{array}{l}\text { No effect on } \\
\text { immediate } \\
\text { learning or } \\
\text { delayed recall }\end{array}$ & & \\
\hline & Crossover design & & Delay: $30 \mathrm{~min}$ & & & \\
\hline \multirow[t]{2}{*}{ Amin et al. (2006) } & 19 menopausal women & $19 F(52)$ & $\begin{array}{l}\text { Verbal paired } \\
\text { associates learning }\end{array}$ & $\begin{array}{l}\text { Impaired } \\
\text { immediate } \\
\text { learning }\end{array}$ & & \\
\hline & Crossover design & & Delay: 30 min & $\begin{array}{l}\text { No effect } \\
\text { on delayed } \\
\text { recall }\end{array}$ & & \\
\hline \multicolumn{7}{|l|}{ Source memory } \\
\hline \multirow[t]{2}{*}{$\begin{array}{l}\text { McAllister-Williams } \\
\text { et al. (2002) }\end{array}$} & 14 healthy volunteers & $14 \mathrm{M}(23)$ & $\begin{array}{l}\text { Episodic Source } \\
\text { Memory Task (EEG) }\end{array}$ & $\begin{array}{l}\text { No effect on } \\
\text { recognition } \\
\text { or response } \\
\text { times }\end{array}$ & & \\
\hline & Crossover design & & Delay: $5 \mathrm{~min}$ & $\begin{array}{l}\text { Impaired } \\
\text { source memory }\end{array}$ & & \\
\hline \multicolumn{7}{|l|}{ General verbal learning } \\
\hline \multirow[t]{2}{*}{ Amin et al. (2006) } & 19 menopausal women & $19 F(52)$ & $\begin{array}{l}\text { Weschler Paragraph } \\
\text { Recall Subtest }\end{array}$ & $\begin{array}{l}\text { Impaired } \\
\text { delayed recall } \\
\text { (immediate } \\
\text { recall results } \\
\text { not reported) }\end{array}$ & & \\
\hline & Crossover design & & Delay: $30 \mathrm{~min}$ & & & \\
\hline \multirow[t]{2}{*}{ Epperson et al. (2007) } & $\begin{array}{l}11 \text { menopausal women } \\
\text { with medicated } \\
\text { depression }\end{array}$ & $11 \mathrm{~F}(50)$ & $\begin{array}{l}\text { Weschler Paragraph } \\
\text { Recall Subtest }\end{array}$ & $\begin{array}{l}\text { No effect on } \\
\text { immediate recall }\end{array}$ & & \\
\hline & Crossover design & & Delay: 30 min & $\begin{array}{l}\text { Impaired delayed } \\
\text { recall }\end{array}$ & & \\
\hline
\end{tabular}

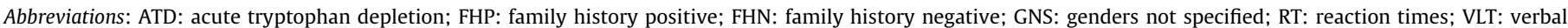

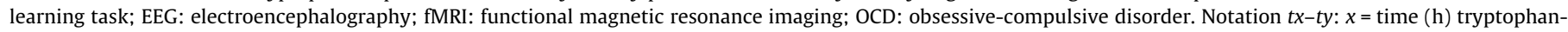
free drink was administered, $y=$ time $(h)$ of testing. 
Table 4

Acute tryptophan depletion studies including non-verbal learning tasks of episodic memory, spatial memory, and semantic memory.

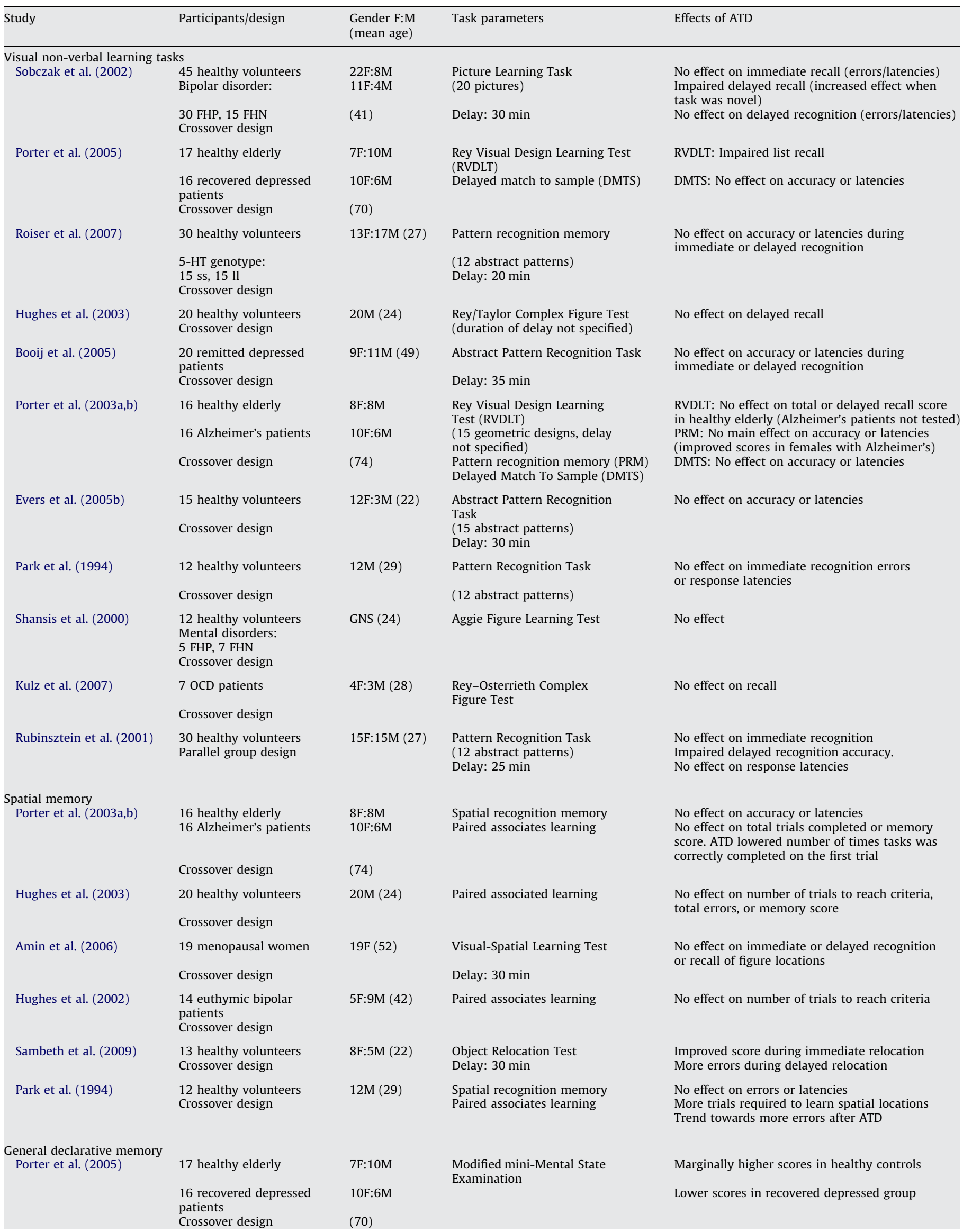


Table 4 (Continued)

\begin{tabular}{|c|c|c|c|c|}
\hline Study & Participants/design & $\begin{array}{l}\text { Gender F:M } \\
\text { (mean age) }\end{array}$ & Task parameters & Effects of ATD \\
\hline \multirow[t]{2}{*}{ Porter et al. (2000) } & 16 healthy elderly & $8 \mathrm{~F}: 8 \mathrm{M}$ & \multirow[t]{2}{*}{$\begin{array}{l}\text { Modified mini-Mental State } \\
\text { Examination }\end{array}$} & No effect in healthy elderly \\
\hline & $\begin{array}{l}16 \text { Alzheimer's patients } \\
\text { Crossover design }\end{array}$ & $\begin{array}{l}\text { 10F:6M } \\
(74)\end{array}$ & & Decreased score in Alzheimer's patients \\
\hline \multirow[t]{2}{*}{ Golightly et al. (2001) } & 25 Schizophrenia patients & \multirow[t]{2}{*}{$4 \mathrm{~F}: 21 \mathrm{M}(45)$} & $\begin{array}{l}\text { Rivermead Behavioral } \\
\text { Memory Test }\end{array}$ & No effect \\
\hline & Crossover design & & $\begin{array}{l}\text { Speed and comprehension of } \\
\text { language processing }\end{array}$ & No effect \\
\hline Park et al. (1994) & $\begin{array}{l}12 \text { healthy volunteers } \\
\text { Crossover design }\end{array}$ & $12 \mathrm{M}(29)$ & Autobiographical Memory Task & No effect \\
\hline \multicolumn{5}{|c|}{ Semantic memory: verbal fluency } \\
\hline Sobczak et al. (2002) & $\begin{array}{l}45 \text { healthy volunteers } \\
\text { Bipolar disorder: } \\
30 \text { FHP, } 15 \text { FHN } \\
\text { Crossover design }\end{array}$ & $\begin{array}{l}22 \mathrm{~F}: 8 \mathrm{M} \\
11 \mathrm{~F}: 4 \mathrm{M} \\
(41)\end{array}$ & Verbal Fluency Test & No effect \\
\hline \multirow[t]{2}{*}{ Porter et al. (2005) } & 17 healthy elderly & 7F:10M & \multirow{2}{*}{$\begin{array}{l}\text { Controlled Oral Word Association } \\
\text { Test } \\
\text { Verbal Fluency } \\
\text { Performance Test }\end{array}$} & No effect \\
\hline & $\begin{array}{l}16 \text { recovered depressed } \\
\text { patients } \\
\text { Crossover design }\end{array}$ & $\begin{array}{l}10 \mathrm{~F}: 6 \mathrm{M} \\
(70)\end{array}$ & & No effect \\
\hline Stewart et al. (2002) & $\begin{array}{l}32 \text { healthy volunteers } \\
\text { Neuroticism: } \\
17 \text { high, } 15 \text { low } \\
\text { Crossover design }\end{array}$ & GNS (22) & Verbal Fluency Test & $\begin{array}{l}\text { Improved performance in low-neuroticism } \\
\text { group } \\
\text { No effect in high-neuroticism group }\end{array}$ \\
\hline Porter et al. (2003a,b) & $\begin{array}{l}16 \text { healthy elderly } \\
16 \text { Alzheimer's patients } \\
\text { Crossover design }\end{array}$ & $\begin{array}{l}8 \mathrm{~F}: 8 \mathrm{M} \\
10 \mathrm{~F}: 6 \mathrm{M} \\
(74)\end{array}$ & Verbal fluency & Results not reported \\
\hline \multirow[t]{2}{*}{ Hughes et al. (2003) } & 20 healthy volunteers & $20 \mathrm{M}(24)$ & $\begin{array}{l}\text { Controlled Oral Word } \\
\text { Association Test }\end{array}$ & $\begin{array}{l}\text { No main effect. ATD increased number } \\
\text { of words produced when task was novel } \\
\text { compared to when task was familiar }\end{array}$ \\
\hline & \multicolumn{3}{|l|}{ Crossover design } & \\
\hline Booij et al. (2005) & $\begin{array}{l}20 \text { remitted depressed } \\
\text { patients } \\
\text { Crossover design }\end{array}$ & $9 \mathrm{~F}: 11 \mathrm{M}(49)$ & $\begin{array}{l}\text { Letter Fluency (Verbal } \\
\text { Fluency Test) }\end{array}$ & $\begin{array}{l}\text { More words produced in first } 30 \mathrm{~s} \\
\text { No effect at } 1 \mathrm{~min}\end{array}$ \\
\hline Luciana et al. (2001) & $\begin{array}{l}19 \text { healthy volunteers } \\
\text { Crossover design }\end{array}$ & GNS (22) & Controlled Word Association Test & No effect of ATD or tryptophan loading \\
\hline Amin et al. (2006) & $\begin{array}{l}19 \text { menopausal women } \\
\text { Crossover design }\end{array}$ & $19 F(52)$ & Controlled Oral Word Association & No effect \\
\hline Merens et al. (2008) & $\begin{array}{l}18 \text { remitted depressed } \\
\text { patients } \\
\text { Crossover design }\end{array}$ & $16 \mathrm{~F}: 2 \mathrm{M}(44)$ & Verbal Fluency Test & No effect \\
\hline Schmitt et al. (2000) & $\begin{array}{l}17 \text { healthy volunteers } \\
\text { Crossover design }\end{array}$ & $9 \mathrm{~F}: 8 \mathrm{M}(23)$ & Verbal Fluency Test & More words produced \\
\hline Gallagher et al. (2003) & $\begin{array}{l}15 \text { healthy volunteers } \\
\text { Crossover design }\end{array}$ & $15 \mathrm{M}(22)$ & Verbal Fluency Test & No effect \\
\hline Allen et al. (2006) & $\begin{array}{l}10 \text { healthy volunteers } \\
\text { Crossover design }\end{array}$ & $\begin{array}{l}2 \mathrm{~F}: 8 \mathrm{M} \\
(23-35)\end{array}$ & Verbal Fluency Task (fMRI) & Results for task performance not reported \\
\hline Morris et al. (1999) & $\begin{array}{l}8 \text { healthy volunteers } \\
\text { Crossover design }\end{array}$ & $8 \mathrm{M}(39)$ & $\begin{array}{l}\text { Paced word repetition task } \\
\text { Orthographic Verbal Fluency } \\
\text { Task (PET) }\end{array}$ & $\begin{array}{l}\text { Results for task performance not reported } \\
\text { Results for task performance not reported }\end{array}$ \\
\hline Smith et al. (1999) & $\begin{array}{l}8 \text { healthy volunteers } \\
\text { Crossover design }\end{array}$ & $8 \mathrm{M}(39)$ & $\begin{array}{l}\text { Paced word repetition task } \\
\text { Orthographic Verbal Fluency Task } \\
\text { (PET) }\end{array}$ & Results for task performance not reported \\
\hline Kulz et al. (2007) & $\begin{array}{l}7 \text { OCD patients } \\
\text { Crossover design }\end{array}$ & $4 \mathrm{~F}: 3 \mathrm{M}(28)$ & $\begin{array}{l}\text { Letter Fluency Test } \\
\text { Five Point Test (non-verbal } \\
\text { fluency) }\end{array}$ & $\begin{array}{l}\text { No effect } \\
\text { No effect }\end{array}$ \\
\hline $\begin{array}{l}\text { Semantic memory primin } \\
\text { Burgund et al. (2003) }\end{array}$ & $\begin{array}{l}16 \text { healthy volunteers } \\
\text { Crossover design }\end{array}$ & $6 \mathrm{~F}: 10 \mathrm{M}(22)$ & Word-Stem Completion Task & $\begin{array}{l}\text { ATD decreased specific-visual priming. } \\
\text { Tryptophan loading decreased amodal } \\
\text { priming }\end{array}$ \\
\hline
\end{tabular}

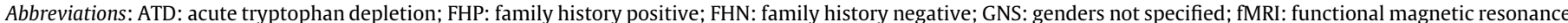
imaging; OCD: obsessive-compulsive disorder. 
Table 5

Acute tryptophan depletion studies including short-term and working memory tasks.

\begin{tabular}{|c|c|c|c|c|}
\hline Study & Participants/design & $\begin{array}{l}\text { Gender F:M } \\
\text { (mean age) }\end{array}$ & Task parameters & Effects of ATD \\
\hline \multicolumn{5}{|l|}{ Short-term memory } \\
\hline Porter et al. (2005) & $\begin{array}{l}17 \text { healthy elderly } \\
16 \text { recovered } \\
\text { depressed patients } \\
\text { Crossover design }\end{array}$ & $\begin{array}{l}\text { 7F:10M } \\
\text { 10F:6M } \\
(70)\end{array}$ & Forward digit span & Impaired in both groups \\
\hline Stewart et al. (2002) & $\begin{array}{l}\text { Neuroticism: } \\
17 \text { high, } 15 \text { low } \\
\text { Crossover design }\end{array}$ & GNS (22) & Forward digit span & No effect \\
\hline Porter et al. (2003a,b) & $\begin{array}{l}16 \text { healthy elderly } \\
16 \text { Alzheimer's patients } \\
\text { Crossover design }\end{array}$ & $\begin{array}{l}8 \mathrm{~F}: 8 \mathrm{M} \\
10 \mathrm{~F}: 6 \mathrm{M} \\
(74)\end{array}$ & Forward digit span & No effect \\
\hline Luciana et al. (2001) & $\begin{array}{l}19 \text { healthy volunteers } \\
\text { Crossover design }\end{array}$ & GNS (22) & $\begin{array}{l}\text { Forward digit span } \\
\text { Spatial span }\end{array}$ & $\begin{array}{l}\text { No effect of ATD or tryptophan loading } \\
\text { No effect of ATD or tryptophan loading }\end{array}$ \\
\hline Shansis et al. (2000) & $\begin{array}{l}12 \text { healthy volunteers } \\
\text { Mental disorders: } \\
5 \text { FHP, } 7 \text { FHN } \\
\text { Crossover design }\end{array}$ & GNS (24) & $\begin{array}{l}\text { Forward digit span } \\
\text { (Hebb's digits) } \\
\text { Corsi blocks }\end{array}$ & $\begin{array}{l}\text { No effect } \\
\text { No effect }\end{array}$ \\
\hline \multicolumn{5}{|l|}{ Verbal working memory } \\
\hline Sobczak et al. (2002) & $\begin{array}{l}45 \text { healthy volunteers } \\
\text { Bipolar disorder: } \\
30 \text { FHP, } 15 \text { FHN } \\
\text { Crossover design }\end{array}$ & $\begin{array}{l}22 \mathrm{~F}: 8 \mathrm{M} \\
11 \mathrm{~F}: 4 \mathrm{M} \\
(41)\end{array}$ & $\begin{array}{l}\text { Sternberg Memory Scanning } \\
\text { Set size: } 1,2 \text {, or } 3\end{array}$ & $\begin{array}{l}\text { Increased overall RT } \\
\text { (tended to decrease RT in FHN group and } \\
\text { not in FHP group) }\end{array}$ \\
\hline Porter et al. (2005) & $\begin{array}{l}17 \text { healthy elderly } \\
16 \text { recovered depressed } \\
\text { patients } \\
\text { Crossover design }\end{array}$ & $\begin{array}{l}7 \mathrm{~F}: 10 \mathrm{M} \\
10 \mathrm{~F}: 6 \mathrm{M} \\
(70)\end{array}$ & Backward digit span & No effect \\
\hline Stewart et al. (2002) & $\begin{array}{l}32 \text { healthy volunteers } \\
\text { Neuroticism: } \\
17 \text { high, } 15 \text { low } \\
\text { Crossover design }\end{array}$ & GNS (22) & $\begin{array}{l}\text { Backward digit span } \\
\text { Paced Auditory Serial Addition } \\
\text { Task }\end{array}$ & $\begin{array}{l}\text { No effect } \\
\text { No effect }\end{array}$ \\
\hline Porter et al. (2003a,b) & $\begin{array}{l}16 \text { healthy elderly } \\
16 \text { Alzheimer's patients } \\
\text { Crossover design }\end{array}$ & $\begin{array}{l}8 \mathrm{~F}: 8 \mathrm{M} \\
10 \mathrm{~F}: 6 \mathrm{M} \\
(74)\end{array}$ & Backward digit span & Impaired in both groups \\
\hline Riedel et al. (1999) & $\begin{array}{l}27 \text { healthy volunteers } \\
\text { Depression: } \\
16 \text { FHP, } 11 \text { FHN } \\
\text { Crossover design }\end{array}$ & $15 \mathrm{~F}: 12 \mathrm{M}(31)$ & $\begin{array}{l}\text { Sternberg Memory Scanning } \\
\text { Set size: } 1,2 \text {, or } 4\end{array}$ & No effect \\
\hline Luciana et al. (2001) & $\begin{array}{l}19 \text { healthy volunteers } \\
\text { Crossover design }\end{array}$ & GNS (22) & Backward digit span & $\begin{array}{l}\text { No effect of ATD. } \\
\text { Tryptophan loading decreased reverse } \\
\text { digit span }\end{array}$ \\
\hline Harrison et al. (2004) & $\begin{array}{l}13 \text { healthy volunteers } \\
\text { Crossover design }\end{array}$ & $13 \mathrm{~F}(22)$ & $\begin{array}{l}\text { Sternberg Memory Scanning } \\
\text { Set size: } 5\end{array}$ & No effect \\
\hline Allen et al. (2006) & $\begin{array}{l}10 \text { Healthy volunteers } \\
\text { Crossover design }\end{array}$ & $\begin{array}{l}2 \mathrm{~F}: 8 \mathrm{M} \\
(23-35)\end{array}$ & $\begin{array}{l}\text { N-back: 0-back and 2-back } \\
\text { (fMRI) }\end{array}$ & No effect on performance (accuracy or RT) \\
\hline \multicolumn{5}{|l|}{ Spatial working memory } \\
\hline Porter et al. (2005) & $\begin{array}{l}17 \text { healthy elderly } \\
16 \text { recovered depressed } \\
\text { patients } \\
\text { Crossover design }\end{array}$ & $\begin{array}{l}\text { 7F:10M } \\
\text { 10F:6M } \\
\text { (70) }\end{array}$ & Spatial working memory & $\begin{array}{l}\text { No effect on within search errors, between } \\
\text { search errors, or strategy use }\end{array}$ \\
\hline Porter et al. (2003a,b) & $\begin{array}{l}16 \text { healthy elderly } \\
16 \text { Alzheimer's patients } \\
\text { Crossover design }\end{array}$ & $\begin{array}{l}8 \mathrm{~F}: 8 \mathrm{M} \\
10 \mathrm{~F}: 6 \mathrm{M} \\
(74)\end{array}$ & Spatial working memory & $\begin{array}{l}\text { No effect on within search errors, between } \\
\text { search errors, or strategy use }\end{array}$ \\
\hline Luciana et al. (2001) & $\begin{array}{l}19 \text { healthy volunteers } \\
\text { Crossover design }\end{array}$ & GNS (22) & Spatial working memory & No effect of ATD or tryptophan loading \\
\hline Harrison et al. (2004) & $\begin{array}{l}13 \text { healthy volunteers } \\
\text { Crossover design }\end{array}$ & $13 F(22)$ & Spatial working memory & $\begin{array}{l}\text { No effect on errors, spatial working memory } \\
\text { index, or response speed }\end{array}$ \\
\hline
\end{tabular}


Table 5 (Continued)

\begin{tabular}{|c|c|c|c|c|}
\hline Study & Participants/design & $\begin{array}{l}\text { Gender F:M } \\
\text { (mean age) }\end{array}$ & Task parameters & Effects of ATD \\
\hline \multirow[t]{2}{*}{ Park et al. (1994) } & 12 healthy volunteers & $12 \mathrm{M}(29)$ & Spatial working memory & $\begin{array}{l}\text { No effect on within search errors, between } \\
\text { search errors, or efficiency of strategy use }\end{array}$ \\
\hline & Crossover design & & & \\
\hline \multicolumn{5}{|c|}{ Affective working memory } \\
\hline Luciana et al. (2001) & $\begin{array}{l}19 \text { healthy volunteers } \\
\text { Crossover design }\end{array}$ & GNS (22) & Affective working memory & $\begin{array}{l}\text { No effect of ATD } \\
\text { Tryptophan loading decreased accuracy } \\
\text { compared to ATD for sad faces only during } \\
\text { trials with longer delays between the target } \\
\text { and probe }\end{array}$ \\
\hline
\end{tabular}

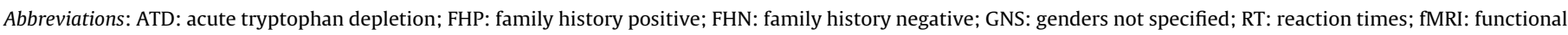
magnetic resonance imaging.

Table 6

Acute tryptophan depletion studies including tasks assessing executive functions.

\begin{tabular}{|c|c|c|c|c|}
\hline Study & Participants/design & $\begin{array}{l}\text { Gender F:M } \\
\text { (mean age) }\end{array}$ & Task parameters & Effects of ATD \\
\hline \multicolumn{5}{|l|}{ Planning } \\
\hline \multirow[t]{3}{*}{ Sobczak et al. (2002) } & 45 healthy volunteers & $22 \mathrm{~F}: 8 \mathrm{M}$ & $\begin{array}{l}\text { One-touch Tower } \\
\text { of London }\end{array}$ & No effect on number of correct responses \\
\hline & Bipolar disorder: & $11 \mathrm{~F}: 4 \mathrm{M}$ & & $\begin{array}{l}\text { Increased response times in the FHP for bipolar } \\
\text { disorder group }\end{array}$ \\
\hline & $\begin{array}{l}30 \mathrm{FHP}, 15 \mathrm{FHN} \\
\text { Crossover design }\end{array}$ & $(41)$ & & \\
\hline \multirow[t]{3}{*}{ Porter et al. (2005) } & 17 healthy elderly & 7F:10M & Tower of London & $\begin{array}{l}\text { No effect on number of excess moves or proportion } \\
\text { of perfect solutions }\end{array}$ \\
\hline & $\begin{array}{l}16 \text { recovered depressed } \\
\text { patients }\end{array}$ & 10F:6M & & \\
\hline & Crossover design & $(68)$ & & \\
\hline \multirow[t]{2}{*}{ Hughes et al. (2003) } & 20 healthy volunteers & $20 \mathrm{M}(24)$ & Tower of London & $\begin{array}{l}\text { No effect on number of excess moves, proportion } \\
\text { of perfect solutions, thinking times, or motor execution } \\
\text { times. Several order effects }\end{array}$ \\
\hline & Crossover design & & & \\
\hline Booij et al. (2005) & $\begin{array}{l}20 \text { remitted depressed } \\
\text { patients } \\
\text { Crossover design }\end{array}$ & $9 \mathrm{~F}: 11 \mathrm{M}(49)$ & Tower of London & $\begin{array}{l}\text { No effect on percentage of correct solutions or reaction } \\
\text { times }\end{array}$ \\
\hline \multirow[t]{2}{*}{ Schmitt et al. (2000) } & 17 healthy volunteers & $9 \mathrm{~F}: 8 \mathrm{M}(23)$ & Tower of London & $\begin{array}{l}\text { No effect on total number of moves or total time } \\
\text { to solve problem }\end{array}$ \\
\hline & Crossover design & & & ATD improved decision-making times \\
\hline \multirow[t]{2}{*}{ Hughes et al. (2002) } & $\begin{array}{l}14 \text { euthymic bipolar } \\
\text { patients }\end{array}$ & $5 F: 9 M(42)$ & Tower of London & $\begin{array}{l}\text { No main effect on proportion of perfect solutions } \\
\text { (trend towards fewer perfect solutions with ATD). } \\
\text { No effect on response times }\end{array}$ \\
\hline & Crossover design & & & \\
\hline \multirow[t]{3}{*}{ Park et al. (1994) } & 12 healthy volunteers & $12 \mathrm{M}(29)$ & Tower of London & $\begin{array}{l}\text { No effect on number of excess moves or efficiency of } \\
\text { planning. }\end{array}$ \\
\hline & Crossover design & & & $\begin{array}{l}\text { Improved initiation times and execution times in naïve } \\
\text { participants }\end{array}$ \\
\hline & & & & $\begin{array}{l}\text { Slowed thinking times in participants already familiar } \\
\text { with the task. }\end{array}$ \\
\hline \multirow[t]{2}{*}{ Murphy et al. (2002) } & & $11 \mathrm{~F}(28)$ & $\begin{array}{l}\text { One-Touch Tower } \\
\text { of London }\end{array}$ & No effect on errors or completion times \\
\hline & Crossover design & & & \\
\hline \multirow[t]{2}{*}{ Kulz et al. (2007) } & 7 OCD patients & 4F:3M (28) & Tower of London & $\begin{array}{l}\text { No significant effects but trends toward impaired } \\
\text { movement times and decreased number of correct } \\
\text { solutions with large effect sizes }\end{array}$ \\
\hline & Crossover design & & & \\
\hline \multicolumn{5}{|l|}{ Decision-Making Tasks } \\
\hline Crean et al. (2002) & $\begin{array}{l}40 \text { healthy volunteers } \\
\text { Alcoholism: } \\
20 \text { FHP, } 20 \text { FHN } \\
\text { Crossover design }\end{array}$ & $40 \mathrm{M}(22)$ & $\begin{array}{l}\text { Delay Discounting Task } \\
\text { (reward preference) }\end{array}$ & $\begin{array}{l}\text { No effect on preference for immediate, smaller rewards } \\
\text { over larger, more delayed rewards }\end{array}$ \\
\hline
\end{tabular}


Table 6 (Continued)

\begin{tabular}{|c|c|c|c|c|}
\hline Study & Participants/design & $\begin{array}{l}\text { Gender F:M } \\
\text { (mean age) }\end{array}$ & Task parameters & Effects of ATD \\
\hline \multirow[t]{4}{*}{ Roiser et al. (2006) } & 30 healthy volunteers & & $\begin{array}{l}\text { Cued-Reinforcement } \\
\text { Reaction Time Task }\end{array}$ & $\begin{array}{l}\text { ATD abolished reinforcement-related speeding in ss } \\
\text { genotype group but not in ll genotype group. ATD sped } \\
\text { response times in ll genotype group. ATD decreased errors } \\
\text { in ss genotype group but had no effect in ll genotype group }\end{array}$ \\
\hline & 5-HT genotype: & $6 \mathrm{~F}: 9 \mathrm{M}$ & & \\
\hline & $15 \mathrm{ss}, 15 \mathrm{ll}$ & $7 F: 8 M$ & & \\
\hline & Crossover design & $(27)$ & & \\
\hline \multirow[t]{2}{*}{ Cools et al. (2005a) } & 22 healthy volunteers & $22 \mathrm{M}(24)$ & $\begin{array}{l}\text { Cued-Reinforcement } \\
\text { Reaction Time Task }\end{array}$ & $\begin{array}{l}\text { ATD slowed RT and increased accuracy during higher } \\
\text { percentage reinforcement trials. ATD impaired ability to } \\
\text { adapt responding to incentive-motivational cues signaling } \\
\text { reinforcement/impaired ability to adapt responding to } \\
\text { incentive-motivational cues signaling reinforcement } \\
\text { certainty }\end{array}$ \\
\hline & $\begin{array}{l}\text { Mixed crossover and } \\
\text { parallel group design }\end{array}$ & & & \\
\hline \multirow[t]{2}{*}{ Rogers et al. (2003) } & 36 healthy volunteers & $9 \mathrm{~F}: 9 \mathrm{M}$ & Decision-Making Task & $\begin{array}{l}\text { ATD group chose 'experimental' gamble when its gains } \\
\text { were large less often than controls. ATD group attenuated } \\
\text { discrimination between different magnitudes of expected } \\
\text { gains. Trend towards decreased deliberation times with } \\
\text { ATD }\end{array}$ \\
\hline & $\begin{array}{l}\text { - ATD }(n=18) \\
\text { - placebo }(n=18) \\
\text { Parallel group design }\end{array}$ & $\begin{array}{l}9 \mathrm{~F}: 9 \mathrm{M} \\
(24)\end{array}$ & & \\
\hline \multirow[t]{3}{*}{ Talbot et al. (2006) } & 32 healthy volunteers & & Decision-Gamble Task & $\begin{array}{l}\text { No effect on speed of decision-making. ATD improved } \\
\text { quality of decision-making; ATD group chose more likely } \\
\text { outcome more often than controls. No effect on risk } \\
\text { taking, delay aversion, and risk adjustment }\end{array}$ \\
\hline & $-\operatorname{ATD}(n=17)$ & $8 \mathrm{~F}: 7 \mathrm{M}$ & & \\
\hline & $\begin{array}{l}\text { - tryptophan loading }(n=15) \\
\text { Parallel group design }\end{array}$ & $\begin{array}{l}8 \mathrm{~F}: 9 \mathrm{M} \\
(34)\end{array}$ & & \\
\hline \multirow[t]{3}{*}{ Rogers et al. (1999b) } & 31 healthy volunteers & & Decision-Making Task & $\begin{array}{l}\text { ATD group chose most likely outcome less often than } \\
\text { controls. Trend towards increased deliberation times. }\end{array}$ \\
\hline & $-\operatorname{ATD}(n=15)$ & & & $\begin{array}{l}\text { No effect on risk adjustment (points put at risk, } \\
\text { impulsivity in selection of bets) }\end{array}$ \\
\hline & $\begin{array}{l}\text { - Placebo }(n=16) \\
\text { Parallel group design }\end{array}$ & $\begin{array}{l}8 \mathrm{~F}: 8 \mathrm{M} \\
(28)\end{array}$ & & \\
\hline \multirow[t]{3}{*}{ Anderson et al. (2003) } & & $11 \mathrm{~F}: 17 \mathrm{M}(23)$ & Gambling-Task & $\begin{array}{l}\text { No effect on the indifference odds-against-winning } \\
\text { for any reward size }\end{array}$ \\
\hline & & & (probabilistic choice) & \\
\hline & Parallel group design & & & \\
\hline \multicolumn{5}{|l|}{ Response inhibition } \\
\hline \multirow[t]{2}{*}{ Clark et al. (2005) } & 42 healthy volunteers & $13 \mathrm{~F}: 29 \mathrm{M}(26)$ & Stop Signal Task & No effect on RT \\
\hline & Crossover design & & & Caused marginally more discrimination errors \\
\hline \multirow[t]{4}{*}{ Crean et al. (2002) } & 40 healthy volunteers & $40 \mathrm{M}(22)$ & Stop Task & No effect on go RT \\
\hline & Alcoholism: & & & $\begin{array}{l}\text { Increased stop RT in FHP group and decreased stop } \\
\text { RT in FHN group }\end{array}$ \\
\hline & 20 FHP, 20 FHN & & & \\
\hline & Crossover design & & & \\
\hline \multirow[t]{3}{*}{ LeMarquand et al. (1998) } & 38 healthy adolescents & $38 \mathrm{M}(17)$ & Go/No-Go Task & No effect \\
\hline & $\begin{array}{l}18 \text { aggressive, } \\
20 \text { non-aggressive }\end{array}$ & & & \\
\hline & Crossover design & & & \\
\hline Evers et al. (2006b) & $\begin{array}{l}13 \text { healthy volunteers } \\
\text { Crossover design }\end{array}$ & $13 \mathrm{M}(23)$ & Go/No-Go Task & No effect on errors or RT \\
\hline \multirow[t]{2}{*}{ Rubia et al. (2005) } & 9 healthy volunteers & GNS (26) & Go/No-Go Task & Decreased errors in choice reaction time during go-process \\
\hline & Crossover design & & & No effect on RT to go signals and omission errors \\
\hline \multirow[t]{2}{*}{ Cools et al. (2005a) } & & $23 \mathrm{M}(24)$ & $\begin{array}{l}\text { Stop-Signal } \\
\text { Reaction-Time Task }\end{array}$ & $\begin{array}{l}\text { No effect on proportion of successful inhibitions or } \\
\text { discrimination error rate }\end{array}$ \\
\hline & $\begin{array}{l}\text { Mixed crossover and } \\
\text { parallel group design }\end{array}$ & & & \\
\hline \multirow[t]{4}{*}{ LeMarquand et al. (1999) } & 57 healthy volunteers & $57 \mathrm{M}(21)$ & Go/No-Go Task & $\begin{array}{l}\text { Increased commission errors in participants with FHP for } \\
\text { alcoholism compared to FHN group }\end{array}$ \\
\hline & Alcoholism: & & & \\
\hline & $24 \mathrm{FHP}, 33 \mathrm{FHN}$ & & & \\
\hline & Parallel g & & & \\
\hline
\end{tabular}

Abbreviations: ATD: acute tryptophan depletion; FHP: family history positive; FHN: family history negative; GNS: genders not specified; RT: reaction times. 
Table 7

Acute tryptophan depletion studies including tasks assessing sustained attention.

\begin{tabular}{|c|c|c|c|c|}
\hline Study & Participants/design & $\begin{array}{l}\text { Gender F:M } \\
\text { (mean age) }\end{array}$ & Task parameters & Effects of ATD \\
\hline \multicolumn{5}{|l|}{ Sustained attention } \\
\hline \multirow[t]{3}{*}{ Porter et al. (2005) } & 17 healthy elderly & $7 F: 10 M$ & Vigil AK & $\begin{array}{l}\text { No effect on delay or commission and omission errors } \\
\text { or response latencies }\end{array}$ \\
\hline & $\begin{array}{l}16 \text { recovered depressed } \\
\text { patients }\end{array}$ & 10F:6M & & \\
\hline & Crossover design & $(70)$ & & \\
\hline \multirow[t]{4}{*}{ Stewart et al. (2002) } & 32 healthy volunteers & GNS (22) & $\begin{array}{l}\text { Visual Change Detection } \\
\text { Task }\end{array}$ & No effect \\
\hline & Neuroticism: & & & \\
\hline & 17 high, 15 low & & & \\
\hline & Crossover design & & & \\
\hline \multirow[t]{2}{*}{ Golightly et al. (2001) } & $\begin{array}{l}25 \text { Schizophrenia } \\
\text { patients }\end{array}$ & $4 \mathrm{~F}: 21 \mathrm{M}(45)$ & Vigil & No effect on response times or omission errors \\
\hline & Crossover design & & & $\begin{array}{l}\text { Trend towards fewer commission errors in first quarter } \\
\text { of test with ATD compared to control }\end{array}$ \\
\hline \multirow[t]{2}{*}{ Walderhaug et al. (2002) } & 24 healthy volunteers & $24 \mathrm{M}(25)$ & CPT - identical pairs & $\begin{array}{l}\text { Lower response criterion (disinhibition) during number } \\
\text { mode }\end{array}$ \\
\hline & Crossover design & & & $\begin{array}{l}\text { Fewer hits and lower perceptual sensitivity during } \\
\text { shape mode }\end{array}$ \\
\hline \multirow[t]{2}{*}{ Walderhaug et al. (2008) } & 24 healthy volunteers & $24 \mathrm{M}(25)$ & CPT -identical pairs & $\begin{array}{l}\text { When task was novel, increased false alarms in number } \\
\text { and shape modes, lower response criterion in number } \\
\text { mode, and decreased perceptual sensitivity in shape mode }\end{array}$ \\
\hline & Crossover design & & & \\
\hline \multirow[t]{2}{*}{ Luciana et al. (2001) } & 19 healthy volunteers & GNS (22) & Letter Cancellation Task & $\begin{array}{l}\text { No main effect on commission errors or completion } \\
\text { times }\end{array}$ \\
\hline & Crossover design & & & $\begin{array}{l}\text { Tryptophan loading led to fewer errors of omission } \\
\text { compared to ATD }\end{array}$ \\
\hline \multirow[t]{3}{*}{ Dougherty et al. (2007) } & 18 healthy volunteers & $14 \mathrm{~F}: 4 \mathrm{M}(27)$ & $\begin{array}{l}\text { Immediate Memory Test } \\
\text { (modified CPT) }\end{array}$ & No effect on correct detections \\
\hline & Crossover design & & & Increased commission errors with ATD \\
\hline & & & & No effect of tryptophan loading on commission errors \\
\hline \multirow[t]{3}{*}{ Porter et al. (2003a,b) } & 16 healthy elderly & $8 \mathrm{~F}: 8 \mathrm{M}$ & Vigil A, Vigil AK & $\begin{array}{l}\text { Vigil K: No effect on errors, response times, response } \\
\text { bias or sensitivity measures }\end{array}$ \\
\hline & Crossover design & $10 \mathrm{~F}: 6 \mathrm{M}$ & & $\begin{array}{l}\text { Vigil AK: Increased commission errors. No effect on } \\
\text { response times, response bias or sensitivity measures }\end{array}$ \\
\hline & & $(74)$ & & \\
\hline \multirow[t]{2}{*}{ Hughes et al. (2002) } & $\begin{array}{l}14 \text { euthymic bipolar } \\
\text { patients }\end{array}$ & $5 F: 9 M(42)$ & Vigil & $\begin{array}{l}\text { No effect on commission or omission errors or response } \\
\text { latencies. Trend towards progressive slowing of RT during } \\
\text { ATD when given on first day }\end{array}$ \\
\hline & Crossover design & & & \\
\hline Harrison et al. (2004) & $\begin{array}{l}13 \text { healthy volunteers } \\
\text { Crossover design }\end{array}$ & $13 \mathrm{~F}(22)$ & Vigilance & No effect on accuracy or RT \\
\hline Sambeth et al. (2009) & $\begin{array}{l}13 \text { healthy volunteers } \\
\text { Crossover design }\end{array}$ & $8 \mathrm{~F}: 5 \mathrm{M}(22)$ & СPT & No effect on accuracies or RT \\
\hline \multirow[t]{2}{*}{ Park et al. (1994) } & 12 healthy volunteers & $12 \mathrm{M}(29)$ & $\begin{array}{l}\text { Rapid visual information } \\
\text { processing }\end{array}$ & No main effect on correct signal detection or response bias \\
\hline & Crossover design & & & $\begin{array}{l}\text { Decreased response latencies when task was novel and } \\
\text { increased response latencies when task was familiar }\end{array}$ \\
\hline \multirow[t]{3}{*}{ Shansis et al. (2000) } & 12 healthy volunteers & GNS (24) & $\begin{array}{l}\text { Mesulam's Cancellation } \\
\text { Task }\end{array}$ & No effect \\
\hline & Mental disorders: & & & \\
\hline & $\begin{array}{l}5 \text { FHP, } 7 \text { FHN } \\
\text { Crossover design }\end{array}$ & & & \\
\hline
\end{tabular}

Abbreviations: ATD: acute tryptophan depletion; FHP: family history positive; FHN: family history negative; GNS: genders not specified; RT: reaction times. 
Table 8

Acute tryptophan depletion studies including tasks assessing focused and divided attention.

\begin{tabular}{|c|c|c|c|c|}
\hline Study & Participants/design & $\begin{array}{l}\text { Gender F:M } \\
\text { (mean age) }\end{array}$ & Task parameters & Effects of ATD \\
\hline \multicolumn{5}{|l|}{ Focused attention } \\
\hline Sobczak et al. (2002) & $\begin{array}{l}45 \text { healthy volunteers } \\
\text { Bipolar disorder: } \\
30 \text { FHP, } 15 \text { FHN } \\
\text { Crossover design }\end{array}$ & $\begin{array}{l}22 \mathrm{~F}: 8 \mathrm{M} \\
11 \mathrm{~F}: 4 \mathrm{M} \\
(41)\end{array}$ & Stroop Colour Word Test & No effect on interference score \\
\hline \multirow[t]{2}{*}{ Hitsman et al. (2007) } & 34 smokers & & Modified Stroop Task & $\begin{array}{l}\text { Increased interference times for all word } \\
\text { cue types (smoking, negative affect, and } \\
\text { positive affect) }\end{array}$ \\
\hline & $\begin{array}{l}\text { - } 19 \text { without history } \\
\text { of depression } \\
\text { - } 15 \text { with history } \\
\text { of depression } \\
\text { Crossover design }\end{array}$ & $\begin{array}{l}6 \mathrm{~F}: 13 \mathrm{M} \\
11 \mathrm{~F}: 4 \mathrm{M} \\
(38)\end{array}$ & & \\
\hline Booij et al. (2005) & $\begin{array}{l}20 \text { remitted depressed } \\
\text { patients } \\
\text { Crossover design }\end{array}$ & $9 \mathrm{~F}: 11 \mathrm{M}(49)$ & $\begin{array}{l}\text { Stroop Colour Word Test } \\
\text { Emotional Stroop Task }\end{array}$ & $\begin{array}{l}\text { Decreased interference score (improved } \\
\text { performance) } \\
\text { Increased interference for positive words } \\
\text { but not negative words }\end{array}$ \\
\hline Horacek et al. (2005) & $\begin{array}{l}20 \text { healthy volunteers } \\
\text { Crossover design }\end{array}$ & 10F:10M (23) & $\begin{array}{l}\text { Stroop Colour Word Test } \\
\text { (fMRI) }\end{array}$ & No effect \\
\hline \multirow[t]{2}{*}{ Schmitt et al. (2000) } & 17 healthy volunteers & 9F:8M (23) & Stroop Colour Word Test & $\begin{array}{l}\text { Decreased interference score (improved } \\
\text { performance) }\end{array}$ \\
\hline & Crossover design & & & \\
\hline \multirow[t]{2}{*}{ Gallagher et al. (2003) } & 15 healthy volunteers & $15 \mathrm{M}(22)$ & Stroop Colour Word Test & $\begin{array}{l}\text { No main effects on latencies or interference } \\
\text { score. Improved performance when ATD } \\
\text { given on second day }\end{array}$ \\
\hline & Crossover design & & & \\
\hline Evers et al. (2006a) & $\begin{array}{l}15 \text { healthy volunteers } \\
\text { Crossover design }\end{array}$ & $15 \mathrm{~F}(22)$ & $\begin{array}{l}\text { Stroop Colour Word Test } \\
\text { Emotional Stroop } \\
\text { (fMRI) }\end{array}$ & $\begin{array}{l}\text { Decreased interference score, no effect } \\
\text { on errors or RT } \\
\text { Increased errors for negative words, no } \\
\text { effect on RT or interference scores }\end{array}$ \\
\hline Scholes et al. (2007) & $\begin{array}{l}12 \text { healthy volunteers } \\
\text { Crossover design }\end{array}$ & $12 \mathrm{M}(26)$ & $\begin{array}{l}\text { Modified Stroop Colour } \\
\text { Word Test }\end{array}$ & $\begin{array}{l}\text { Decreased interference score (improved } \\
\text { performance) } \\
\text { No effect on response times }\end{array}$ \\
\hline \multirow[t]{3}{*}{ Munafo et al. (2006) } & 24 healthy controls & $14 \mathrm{~F}: 10 \mathrm{M}$ & Emotional Stroop Task & $\begin{array}{l}\text { Slowed colour naming for socially threatening } \\
\text { stimuli compared to tryptophan loading in } \\
\text { recovered depressed patients on medication }\end{array}$ \\
\hline & Depression: & $14 \mathrm{~F}: 10 \mathrm{M}$ & & $\begin{array}{l}\text { No effect in healthy controls or unmedicated } \\
\text { recovered depressed }\end{array}$ \\
\hline & $\begin{array}{l}24 \text { remitted off medication } \\
24 \text { remitted on medication } \\
\text { Parallel group design }\end{array}$ & $\begin{array}{l}14 \mathrm{~F}: 10 \mathrm{M} \\
(37)\end{array}$ & & \\
\hline Hayward et al. (2005) & $\begin{array}{l}24 \text { healthy controls } \\
24 \text { recovered depressed } \\
\text { patients } \\
\text { Parallel group design }\end{array}$ & $\begin{array}{l}\text { 14F:10M } \\
14 \mathrm{~F}: 10 \mathrm{M} \\
(38)\end{array}$ & $\begin{array}{l}\text { Counting Stroop } \\
\text { Emotional Counting Stroop }\end{array}$ & $\begin{array}{l}\text { No effect during Counting Stroop } \\
\text { Increased emotional interference score } \\
\text { during Emotional Counting Stroop }\end{array}$ \\
\hline \multicolumn{5}{|c|}{ Focused and divided attention } \\
\hline Sobczak et al. (2002) & $\begin{array}{l}45 \text { healthy volunteers } \\
\text { Bipolar disorder: } \\
30 \text { FHP, } 15 \text { FHN } \\
\text { Crossover design }\end{array}$ & $\begin{array}{l}22 \mathrm{~F}: 8 \mathrm{M} \\
11 \mathrm{~F}: 4 \mathrm{M} \\
(41)\end{array}$ & $\begin{array}{l}\text { Dichotic Listening Task: } \\
\text { - Focused Attention Subtask } \\
\text { - Divided Attention Subtask }\end{array}$ & $\begin{array}{l}\text { No effect on focused attention } \\
\text { No effect on divided attention }\end{array}$ \\
\hline Schmitt et al. (2000) & $\begin{array}{l}17 \text { healthy volunteers } \\
\text { Crossover design }\end{array}$ & $9 \mathrm{~F}: 8 \mathrm{M}(23)$ & $\begin{array}{l}\text { Dichotic Listening Task: } \\
\text { - Focused Attention Subtask } \\
\text { - Divided Attention Subtask }\end{array}$ & $\begin{array}{l}\text { Improved focused attention. } \\
\text { No effect on divided attention }\end{array}$ \\
\hline
\end{tabular}

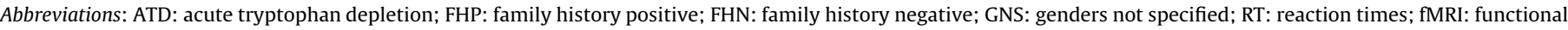
magnetic resonance imaging.

The ATD studies included in the review are summarized in Tables 2-10. The tables are organized according to tasks measuring specific cognitive domains and functions. Within each cognitive domain, the studies are approximately ordered according to design power; within-subjects studies are ordered first, followed by mixed and between-subjects designs and those with higher numbers of participants appear earlier.

\section{Results and discussion}

\subsection{Psychomotor processing}

Psychomotor processing involves fundamental cognitive operations that enable sensation, perception and motor actions (Lezak, 2004). Serotonin may contribute to psychomotor proces- 
Table 9

Acute tryptophan depletion studies including tasks assessing attentional set-shifting and reversal learning.

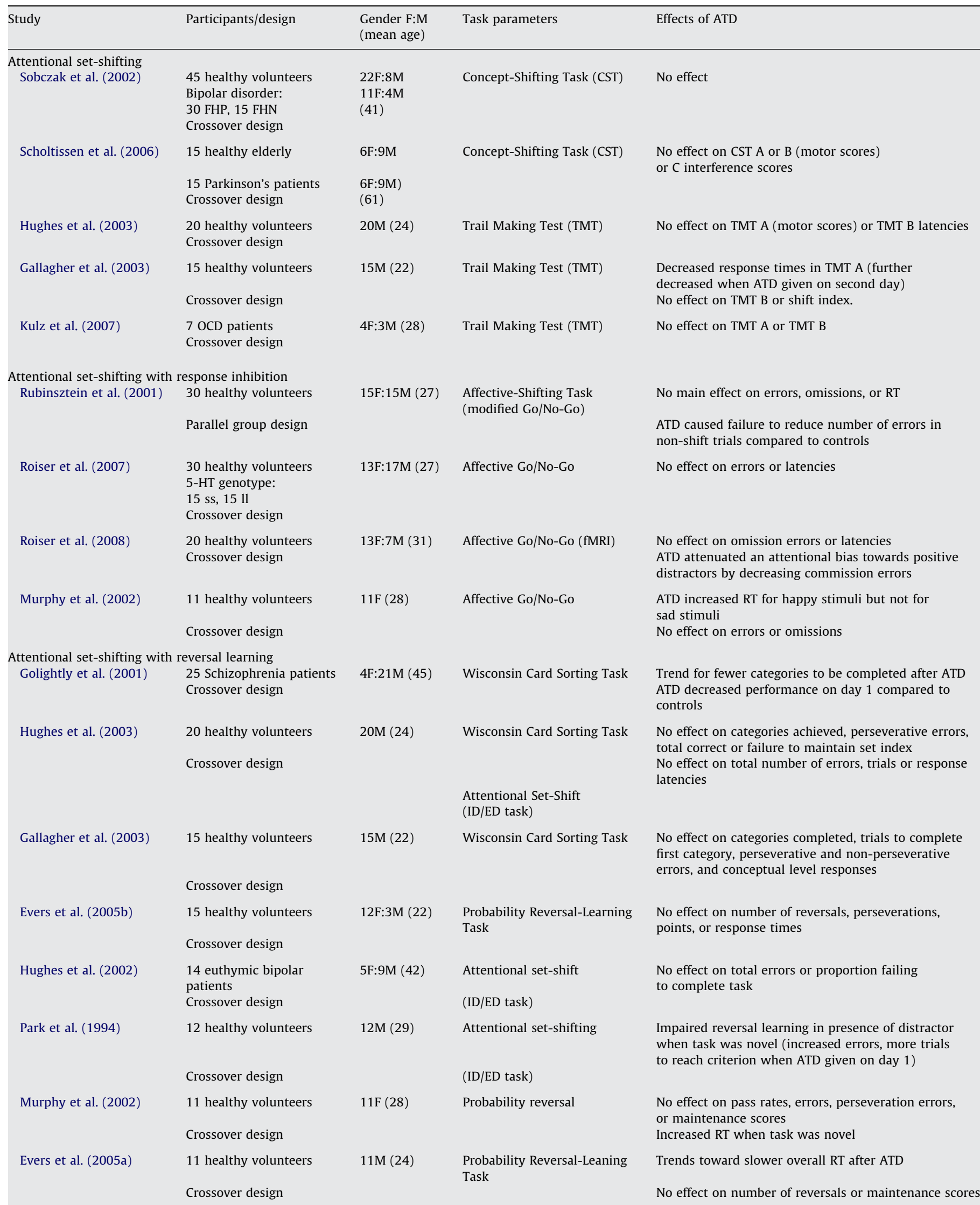


Table 9 (Continued)

\begin{tabular}{|c|c|c|c|c|}
\hline Study & Participants/design & $\begin{array}{l}\text { Gender F:M } \\
\text { (mean age) }\end{array}$ & Task parameters & Effects of ATD \\
\hline Rogers et al. (1999a) & $\begin{array}{l}55 \text { healthy volunteers } \\
\text { - ATD }(n=15) \\
\text { - placebo }(n=16) \\
\text { Parallel group design }\end{array}$ & $\begin{array}{l}8 \mathrm{~F}: 7 \mathrm{M} \\
8 \mathrm{~F}: 32 \mathrm{M} \\
(26)\end{array}$ & $\begin{array}{l}\text { Attentional set shift } \\
\text { (ID/ED task) }\end{array}$ & $\begin{array}{l}\text { Fewer subjects completed task beyond the CDR stage } \\
\text { ATD increased errors } \\
\text { ATD produced a deficit in learning a reversal shift } \\
\text { No effect on response latencies }\end{array}$ \\
\hline \multirow[t]{2}{*}{ Talbot et al. (2006) } & 32 healthy volunteers & & Attentional set-shift & $\begin{array}{l}\text { No effect of ATD or tryptophan loading on number } \\
\text { completing task, numbers of trials completed, error } \\
\text { rates or deliberation times }\end{array}$ \\
\hline & $\begin{array}{l}\text { - tryptophan loading } \\
(n=15) \\
\text { - ATD }(n=17) \\
\text { Parallel group design }\end{array}$ & $\begin{array}{l}8 \mathrm{~F}: 7 \mathrm{M} \\
8 \mathrm{~F}: 9 \mathrm{M} \\
(34)\end{array}$ & (ID/ED task) & \\
\hline
\end{tabular}

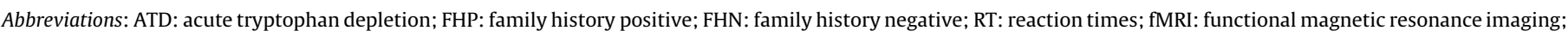
ID/ED: intra-dimensional/extra-dimensional shift; OCD: obsessive-compulsive disorder.

Table 10

Acute tryptophan depletion studies including tasks assessing responses to emotional stimuli.

\begin{tabular}{|c|c|c|c|c|}
\hline Study & Participants/design & $\begin{array}{l}\text { Gender F:M } \\
\text { (mean age) }\end{array}$ & Task parameters & Effects of ATD \\
\hline \multicolumn{5}{|c|}{ Emotional information processing } \\
\hline \multirow[t]{4}{*}{$\begin{array}{l}\text { van der Veen } \\
\text { et al. (2007) }\end{array}$} & 24 healthy volunteers & $24 \mathrm{~F}(23)$ & $\begin{array}{l}\text { Morphed Facial Expression and } \\
\text { Gender Categorization Task (fMRI) }\end{array}$ & ATD effects on accuracy explained as an order effect \\
\hline & Depression & & & \\
\hline & 13 FHP, 11 FHN & & & \\
\hline & Crossover design & & & \\
\hline \multirow[t]{4}{*}{ Merens et al. (2008) } & $\begin{array}{l}18 \text { remitted depressed } \\
\text { patients }\end{array}$ & $16 \mathrm{~F}: 2 \mathrm{M}(44)$ & Facial Expression Recognition Task & Decreased accuracies for fear recognition \\
\hline & Crossover design & & Dot Probe Test & Sped recognition times for faces expressing disgust \\
\hline & & & Implicit Association Test & Dot Probe: No effect \\
\hline & & & & Implicit Association Test: No effect \\
\hline \multirow[t]{2}{*}{ Cools et al. (2005b) } & 12 healthy volunteers & $12 \mathrm{M}(24)$ & $\begin{array}{l}\text { Gender Categorization Task with } \\
\text { facial expression recognition (fMRI) }\end{array}$ & $\begin{array}{l}\text { No behavioral effects (proportion of correct } \\
\text { responses) }\end{array}$ \\
\hline & Crossover design & & & \\
\hline \multirow[t]{2}{*}{ Fusar-Poli et al. (2007) } & 10 healthy volunteers & 2F:10M (26) & $\begin{array}{l}\text { Gender Categorization Task with } \\
\text { facial expression recognition (fMRI) }\end{array}$ & No effect on accuracies or response latencies \\
\hline & Crossover design & & & \\
\hline \multirow[t]{2}{*}{ Williams et al. (2007) } & 10 healthy volunteers & $10 \mathrm{M}(18-30)$ & $\begin{array}{l}\text { Facial Expression Recognition Task } \\
\text { with face-direction conditions (fMRI) }\end{array}$ & Decreased response latencies \\
\hline & Crossover design & & & $\begin{array}{l}\text { Increased errors for side-viewed compared to } \\
\text { front-viewed faces }\end{array}$ \\
\hline \multirow[t]{4}{*}{ Hayward et al. (2005) } & 24 healthy controls & $14 \mathrm{~F}: 10 \mathrm{M}$ & Facial expression recognition & $\begin{array}{l}\text { Improved accuracy for recognition of happy facial } \\
\text { expressions in controls and impaired recognition } \\
\text { of happy facial expressions in recovered depressed } \\
\text { patients }\end{array}$ \\
\hline & $\begin{array}{l}24 \text { recovered depressed } \\
\text { patients }\end{array}$ & 14F:10M & & $\begin{array}{l}\text { EPS: No effect in healthy controls. Elevation of } \\
\text { startle response in recovered depressed patients }\end{array}$ \\
\hline & Parallel group design & $(38)$ & & \\
\hline & & & Emotional potential startle (EPS) & \\
\hline \multirow[t]{2}{*}{ Harmer et al. (2003) } & 38 healthy volunteers & $18 \mathrm{~F}: 20 \mathrm{M}(24)$ & Facial Expression Recognition Task & $\begin{array}{l}\text { ATD decreased accuracy for recognition of fearful } \\
\text { faces in females but not males. ATD slowed } \\
\text { responses to fearful faces in males and females }\end{array}$ \\
\hline & Parallel group design & & & No effect on judgments of non-emotional faces \\
\hline \multirow[t]{4}{*}{ Marsh et al. (2006) } & & $14 \mathrm{~F}: 12 \mathrm{M}(28)$ & Morphed Facial Expression Task & $\begin{array}{l}\text { ATD decreased accuracy for recognition of fearful } \\
\text { faces in sl heterozygotes but not ll homozygotes }\end{array}$ \\
\hline & 5-HT genotype & & & No effect on response latencies \\
\hline & $15 \mathrm{sl}, 11 \mathrm{ll}$ & & & \\
\hline & Parallel group design & & & \\
\hline
\end{tabular}

Abbreviations: ATD: acute tryptophan depletion; FHP: family history positive; FHN: family history negative; fMRI: functional magnetic resonance imaging.

sing through pathways ascending from the raphe nuclei to the cerebellum, the cerebral cortex, and the striatum (Nieuwenhuys, 1985; Tork, 1990). If 5-HT is an integral neurotransmitter underlying psychomotor performance, then ATD may induce psychomotor agitation and retardation.

Evidence from electroencephalography studies suggests ATD alters the electrophysiological activity in the brain underlying stimulus processing. Attentional processes are accompanied by characteristic components in event-related potentials (Pakarinen et al., 2007). Attention to a tone enhances an early P50 component and selective attention corresponds with a processing negativity comprised of an earlier negative peak (N1) generated around the auditory cortex; subsequent features of the processing negativity are related to prefrontal activity (Ahveninen et al., 2003). During a 
selective attention task, ATD delayed P50 latencies and suppressed the P50 and N1 amplitudes but left the later processing negativity components associated with prefrontal activity unaffected (Ahveninen et al., 2003). Similarly, ATD altered early processing components related to stimulus change detection and did not affect later components (Ahveninen et al., 2002). During an oddball task requiring responding to stimuli presented infrequently, ATD increased the amplitudes and decreased the latencies of the mismatch negativity, a component reflecting preattentive detection of stimulus changes in the auditory cortex (Kahkonen et al., 2002). Although effects on performance were not reported in any of these studies, ATD altered brain responses to auditory stimuli believed to underlie early cortical processing of auditory stimuli supporting serotonin's role in early perceptual processing.

Tasks designed to assess psychomotor processing can be categorized into tests that primarily tap perceptual functioning, motor abilities, or both. Tasks assessing perceptual functions typically require the detection of visually presented targets or signals. Several studies investigated the effects of ATD on visual search and discrimination (Table 2). Park et al. (1994) had participants detect consecutive even or odd digits in an array of digits; ATD did not alter perceptual sensitivity, response bias, or response latencies overall. When the task was novel, ATD decreased response latencies and when the task was familiar, ATD increased response latencies, but these findings were attributed to impedance of information retrieval. In another study, participants responded to an array of shifting dots when four dots formed a square; ATD did not affect perceptual sensitivity or reaction times (Riedel et al., 1999). Similarly, Harrison et al. (2002) found no effect of ATD on visual discrimination during an inspection time task which required participants to identify the short or long lines in an image followed by backward-masking. Stewart et al. (2002) also reported no effect of ATD during a visual change detection task. Another two studies employed continuous performance tasks tapping psychomotor processing. ATD reduced the perceptual sensitivity and response bias for shapes and numbers, respectively but the authors concluded that ATD leads to an impulsive response style for verbal stimuli and that ATD reduces attention for spatial stimuli (Walderhaug et al., 2002, 2008). Thus although some treatment effects were noted in both these studies, the results were not interpreted as evidence that ATD directly affects psychomotor processing. Alternatively, in one study ATD improved visual discrimination abilities during a critical flicker-fusion threshold task requiring responses when a flickering red light appeared continuous (Harrison et al., 2004). Overall, ATD does not appear to impair sensory processing abilities and may improve some aspects of visual perception.

Several tasks designed to assess psychomotor processing involve visual and motor components including the digit-symbol substitution test (DSST) and reaction time (RT) tasks (Table 2). The DSST involves writing digits into an array of empty boxes positioned below symbols according to a coding table indicating the correspondence between digits and symbols. ATD did not affect completion times during the DSST in one study (Schmitt et al., 2000) but ATD improved performance on the DSST in a sample of adults classified as having high or low neuroticism (Stewart et al., 2002). One possible explanation for these contradictory findings is that Stewart et al. (2002) selected participants according to extreme scores on a neuroticism scale while Schmitt et al. (2000) randomly recruited healthy volunteers. With respect to RT tasks, ATD did not affect simple RT in healthy volunteers (Harrison et al., 2004); however, ATD improved simple RT and RT in a fingerprecuing task in both healthy elderly participants and patients with Parkinson's disease (Scholtissen et al., 2006). Motor choice RT is a more complicated task requiring participants to vary responses according to the stimulus presented. ATD did not affect response latencies in motor choice RT tasks in healthy adults or recovered depressed patients (Booij et al., 2005; Harrison et al., 2004; Riedel et al., 1999; Schmitt et al., 2000). The Concept-Shifting Task and the Trail Making Task include preliminary trials that assess psychomotor speed. Of the five studies employing one of these tasks, four found no effect of ATD (Hughes et al., 2003; Kulz et al., 2007; Scholtissen et al., 2006; Sobczak et al., 2002) and one study reported that ATD improved psychomotor speed (Gallagher et al., 2003).

The last type of psychomotor tasks involves predominantly motor abilities (Table 2). ATD did not effect motor abilities during finger tapping or the manipulation of pegs in a grooved pegboard although TRP loading caused participants to drop more pegs (Luciana et al., 2001). The authors attributed the detrimental effect on fine motor coordination to the concept that elevated 5-HT restricts information flow causing fine motor deficits. In a motor screening task, ATD did not impair the ability to touch a screen presenting a flashing cross that appeared in different positions in both healthy elderly or patients with Alzheimer's disease (Porter et al., 2003b). In summary, the majority of findings suggest that ATD does not directly impair either perceptual processing or psychomotor abilities.

\subsection{Declarative memory}

\subsubsection{Episodic memory}

4.2.1.1. Verbal learning task. Declarative memory involves the acquisition and retention of information demanding conscious or explicit learning (Lezak, 2004; Squire, 1987). Episodic memory is memory for events and experiences and semantic memory is memory for factual knowledge (Squire, 1987; Tulving, 1983, 1992). Episodic memory tasks typically involve three phases; encoding, consolidation and retrieval (Lezak, 2004). During the encoding phase, the information presented is acquired and learned. Consolidation is the processing of encoded information into long-term storage for later retrieval. Stored information is then either recognized through prompting or recalled spontaneously during the retrieval phase. The majority of ATD studies on cognition have utilized tasks assessing episodic memory function such as verbal learning tasks (VLT), non-verbal learning tasks, and spatial memory tasks. Given serotonin's projection to the hippocampus (Tork, 1990), we hypothesize that ATD impairs aspects of episodic memory functioning.

Word learning tasks can assess short-term memory (immediate recall), long-term episodic memory and consolidation (delayed recall and recognition). Eight studies investigated the effects of ATD using the visual VLT (Table 3). Generally, these studies differed with respect to the number of words to be learned and the number of times the lists were presented. However, the results were relatively consistent; ATD impaired delayed recall, but not immediate recall. Riedel et al. (1999) and Schmitt et al. (2000) concluded that ATD affects specifically memory consolidation because delayed recall is only impaired when learning occurs in a tryptophan-depleted state. In contrast, when participants learn the word lists before depletion, delayed recall remains intact (Riedel et al., 1999; Schmitt et al., 2000).

Several variations of the visual VLT were utilized in ATD studies. The auditory VLT involves the oral presentation of a 15 word list. Of the seven ATD studies employing the auditory VLT, four found no effect on immediate and delayed recall in healthy adults, elderly, or patients with Alzheimer's disease (Hayward et al., 2005; Hughes et al., 2003; Porter et al., 2003b; Shansis et al., 2000) and four reported no effect on delayed recognition in healthy adults, elderly, and patients with recovered depression or OCD (Hayward et al., 2005; Hughes et al., 2003; Kulz et al., 2007; Porter et al., 2003b) 
(Table 3). In contrast, one study found impaired immediate and delayed recall following ATD in healthy volunteers (Merens et al., 2008), although most of the volunteers were female and the effects of ATD on VLT performance are more pronounced in females (Sambeth et al., 2007). Similarly, Hayward et al. (2005) reported that recovered depressed patients exhibited impaired immediate recall and another study found ATD impaired delayed recall in healthy elderly and recovered depressed patients (Porter et al., 2005). In general, the ATD studies utilizing the auditory VLT produced results that are inconsistent with the visual VLT findings for several possible reasons. Some of these studies used different designs or unique samples. Hayward et al. (2005) employed a between-subjects design for treatment and Kulz et al. (2007) tested only seven OCD patients; these differences may have resulted in underpowered negative findings. Hughes et al. (2003) tested males only and males are known to be less affected by ATD (Sambeth et al., 2007) and the Porter et al. (2005) tested elderly patients who may be more vulnerable to ATD effects. The use of a 15 word list may have introduced a ceiling effect. It is also possible that the auditory modality is differentially affected by ATD. A single study testing both the auditory and visual VLT in the same participants may help resolve this discrepancy.

A further modification of the VLT is the affective VLT which includes words with emotional valences. Two studies revealed that ATD impaired delayed recall of neutral and positive words, but not of negative words (Kilkens et al., 2004; Klaassen et al., 2002) and immediate recall was also impaired in one study (Kilkens et al., 2004) but not the other (Klaassen et al., 2002) (Table 3). The lack of effect on negative words evidenced mood congruent memory bias, although the ATD effect on recall was not systematically related to changes in mood induced by ATD (Klaassen et al., 2002). Using a variation of the affective VLT, Hayward et al. (2005) found no effect on immediate recall. Roiser et al. (2007) used an affective directed forgetting paradigm where participants are informed which words are to be remembered or forgotten although all words are tested during a recognition test. ATD only impaired immediate recall in participants with a 5-HT transporter genotype imparting vulnerability for affective disorders (ss genotype of the 5-HT transporter polymorphism) and did not affect delayed recognition in both the participant groups. This finding adheres to the principle that the effects of ATD are more pronounced in populations with serotonergic vulnerability.

A functional magnetic resonance imaging (fMRI) study that included an affective word learning task produced results consistent with some of these findings. During encoding, participants rated the words as positive, negative or neutral and were aware that recall would be tested later. Interestingly, ATD led to more words being rated as positive but decreased delayed recognition for positive words. Functionally, ATD decreased activation in the right hippocampus during encoding but not during retrieval (van der Veen et al., 2006). The authors concluded that memory consolidation may begin at the time of encoding and ATD may impair the consolidation as early as at the time of encoding. All in all, the results from the affective VLT are largely consistent with the visual VLT results suggesting that ATD also influences the delayed recall of emotionally loaded words.

The above summary demonstrates that the most consistent outcome of ATD studies involving the VLT is that when learning occurs in a tryptophan-depleted state, immediate recall of words remains intact while delayed recall is impaired. However, several of the studies did show (non-)significant decrements in immediate recall (e.g., Kilkens et al., 2004; Porter et al., 2005; Schmitt et al., 2000; Scholtissen et al., 2006). To increase the power of the immediate recall scores, Sambeth et al. (2007) performed a 'mega-analysis' by pooling data from nine ATD studies. ATD significantly impaired immediate recall but women were affected more than men. The authors concluded that ATD affects encoding as well as impairing consolidation; however, to a lesser extent than consolidation because the slope of the learning curve was unaffected by ATD.

4.2.1.2. Other tests of episodic memory. Several other studies investigated the effects of ATD on episodic memory with tasks involving verbal learning (Table 3). Two studies presented participants with paragraphs to be recalled immediately and after a 30 min delay. ATD significantly impaired delayed recall of the paragraphs (Amin et al., 2006; Epperson et al., 2007). In the verbal paired associated learning task (vPAL), participants remember eight pairs of words and must complete the pair when prompted after a 30 min delay. ATD did not affect immediate learning or delayed recall during the VPAL in healthy volunteers (Hughes et al., 2003). In contrast, ATD impaired immediate learning but not delayed recall in healthy menopausal women (Amin et al., 2006). These equivocal findings may be explained by difference in the same populations tested; Hughes et al. (2003) tested young males while Amin et al. (2006) included only menopausal women (women are known to be more sensitive to the effects of ATD on episodic memory, Sambeth et al., 2007).

Source memory involves the recollection of contextual information for episodic memories. Using an elegant design, McAllisterWilliams et al. (2002) explored the effects of ATD on episodic source memory with a word learning task (Table 3). Participants learned words presented verbally by either a female or male voice. Recognition was later tested through judgments of word familiarity and source memory was assessed by having participants recall the gender of the voice that presented the word. Interestingly, ATD did not affect recognition; however, ATD significantly impaired source memory recall. The memory impairment was not associated with changes in the magnitude or topography of event-related potentials measured with electroencephalography.

Several ATD studies attempted to extend the findings from the VLT to non-verbal learning (Table 4). Eleven studies utilized the Pattern Recognition Memory Task or similar variations in which participants identified familiar or new patterns. Generally, no effect on immediate or delayed recognition was observed, except in the studies of Rubinsztein et al. (2001), Sobczak et al. (2002) and Porter et al. (2005), who found impaired delayed recall or recognition. The high accuracies observed in these studies suggest that picture learning is easier than verbal learning and this may explain the lack of effects. Although, the majority of the studies converge to indicate that ATD does not affect non-verbal visual learning, some evidence exists that memory consolidation deficits caused by ATD may not be modality specific (Rubinsztein et al., 2001).

A distinct type of episodic memory is spatial memory; the acquisition and recall of information pertaining to the spatial layout of an environment (Barnes, 1988). Of the six studies testing the effects of ATD on visual spatial memory, ATD did not influence immediate recognition in three (Amin et al., 2006; Park et al., 1994; Porter et al., 2003b) (Table 4). In one study, ATD improved immediate spatial recall and impaired delayed spatial recall, a result consistent with the visual VLT findings (Sambeth et al., 2009). In contrast, discrepant results were obtained from a paired associates learning task where participants recognized the locations of abstract patterns after a short delay. Park et al. (1994) found that ATD increased the number of trials required to learn the locations of the abstract patterns in healthy adults while three studies found no effect in healthy adults, healthy elderly, patients with Alzheimer's disease or euthymic bipolar patients (Hughes et al., 2002, 2003; Porter et al., 2003b). Given that only two studies reported contradictory outcomes within this domain, it appears that ATD does not impair spatial memory. 
Lastly, other tasks assessing general declarative memory were included in several ATD studies (Table 4). Park et al. (1994) assessed recall for autobiographical information and found that ATD did not affect recall. In patients with Alzheimer's disease and recovered depressed patients, ATD decreased scores on the Modified Mini-Mental State Exam, but not in healthy elderly (Porter et al., 2000, 2005). Golightly et al. (2001) administered the Rivermead Behavioral Memory Test designed to assess memory problems in daily living to a group of Schizophrenics and found no effect of ATD. Similarly, ATD did not impair performance on the Speed and Comprehension of Language Processing Test (Golightly et al., 2001).

To summarize, ATD effects on episodic memory are most robust for the visual VLT; ATD impairs the consolidation of verbal information and encoding to a lesser extent. ATD also impairs the recall of source episodic memory. The findings are less consistent for auditory VLT and non-verbal learning tasks. ATD does not appear to affect spatial episodic memory or general declarative memory when assessed with non-specific tasks.

\subsubsection{Semantic memory}

Semantic memory involves the learning of factual knowledge (Squire, 1987). The most common assessment of semantic memory is the verbal fluency task where participants list off as many words possible beginning with a certain letter. Verbal fluency tasks are considered a measure of strategy driven retrieval from semantic memory stores and thus they also demand executive functions. Existing evidence converges to indicate that ATD does not impair semantic memory in healthy participants. Of the 15 studies that examined ATD effects on verbal fluency (Table 4), 10 reported that ATD did not impair semantic memory (Allen et al., 2006; Amin et al., 2006; Gallagher et al., 2003; Hughes et al., 2003; Kulz et al., 2007; Luciana et al., 2001; Merens et al., 2008; Morris et al., 1999; Porter et al., 2005; Sobczak et al., 2002). However, a pooled analysis of two studies (Porter et al., 2003b, 2005) revealed that ATD impaired verbal fluency in healthy elderly (Mace et al., 2008). In contrast, two studies reported ATD improved verbal fluency performance in healthy adults and individuals who scored low on neuroticism scales (Schmitt et al., 2000; Stewart et al., 2002) and in another study improvements in verbal fluency in recovered depressed patients were noted in the first $30 \mathrm{~s}$ that disappeared at $1 \mathrm{~min}$ (Booij et al., 2005).

Three neuroimaging studies investigated the effects of ATD on verbal fluency using a pace word repetition task, where participants immediately repeat a word presented by the experimenter, and a paced orthographic fluency task, where participant generate a word beginning with a certain letter. ATD did not affect performance on either task (Morris et al., 1999; Smith et al., 1999). However, in one PET study involving participants with histories of depression, ATD decreased activation in the left amygdala and left anterior cingulate cortex during the verbal fluency task compared to the word repetition task (Morris et al., 1999). Previous research had implicated these regions in language generation tasks and mood disorders supporting the concept that depleted 5-HT contributes to the language-related deficits seen in depression. Consistent with this assertion, another study using the same dataset showed that mood response to ATD covaried with activity in several regions including the anterior cingulate and orbitofrontal cortices (Smith et al., 1999). Lastly, an fMRI study involving healthy participants found that ATD decreased taskspecific activation in the left medial frontal gyrus and in the precuneus during a verbal fluency task and these changes were unrelated to mood response (Allen et al., 2006). Together, these findings suggest that mood and functional alterations in brain circuitry may interact to contribute to the cognitive impairments associated with depression.
Only one other study has investigated semantic memory using a word-completion task that assessed priming effects (Burgund et al., 2003) (Table 4). ATD decreased the influence of specifically visual priming cues and TRP loading decreased amodal priming effects. This study highlights the importance of assessing the effects of ATD on semantic memory with more than one type of task. In the future, several assessments of semantic memory functioning should be utilized for examining the effects of ATD in this domain to provide a more comprehensive perspective.

In summary, most studies reported ATD did not affect semantic memory, some showed an improvement, and a pooled analysis found that ATD impaired semantic memory in healthy elderly. Taken together, the findings suggest that ATD does not impair or may improve semantic memory in healthy adults. Future studies should include tasks other than verbal fluency to further clarify serotonin's role in semantic memory.

\subsection{Working memory}

Working memory is generally considered to involve the brief online storage and manipulation of stored information (Baddeley, 1998; Cowan, 2008). Thus working memory operations involve a short-term memory storage component and executive functions (to be addressed later). ATD effects on short-term memory will be reviewed prior to summarizing research using tasks that involve both the short-term storage and manipulation component of working memory.

Short-term memory involves the storage of information acquired from sensory input or through mental processing for brief periods of time (Cowan, 2008). Therefore, tasks assessing short-term memory function also depend on an immediate attention component. Forward and backward digit span are one method for dissociating the short-term memory component and the executive function component of working memory. Forward digit span only requires brief storage of items to be recalled immediately after presentation whereas backwards digit span demands both the storage of items and information manipulation. Of the five studies that investigated the effects of ATD on shortterm memory with forward digit span, four reported no effect in healthy adults, healthy elderly, participants with high or low neuroticism scores, and patients with Alzheimer's disease (Luciana et al., 2001; Porter et al., 2003b; Shansis et al., 2000; Stewart et al., 2002) (Table 5). TRP loading also did not alter forward digit span in healthy adults (Luciana et al., 2001). In contrast, Porter et al. (2005) found ATD impaired forward digit span in healthy elderly and elderly patients with remitted depression, a finding they attributed to the working memory deficits accompanying aging. The same group previously found no effect in healthy elderly or Alzheimer's patients on the same task (Porter et al., 2003b). Different dosages of ATD were used in the two studies and a pooled analysis of both studies reported that ATD impaired forward digit span in females under higher dose (100 g amino acid drink) (Mace et al., 2008). Shansis et al. (2000) tested short-term memory using Corsi's blocks, a non-verbal analogue of digit span and found no effect of ATD. Similarly, Luciana et al. (2001) measured spatial span by having participants repeat a sequence of lit boxes and reported no effect of ATD or TRP loading. Thus, the majority of the findings from ATD studies investigating short-term memory functioning suggest ATD does not impair short-term memory for verbal and non-verbal information. This conclusion does not fit with the findings from the ATD studies that included the VLT which found an effect of ATD on immediate recall, an indicator of short-term memory abilities (Sambeth et al., 2007). The majority of VLT studies used 30 word lists and the digit span tasks only tested a maximum of ten digits and therefore the VLT may be more sensitive to the effects of ATD on short-term memory due to increased task demand. Given that 
ATD does not appear to affect short-term memory involving relatively low numbers of stored items, any potential deficits in working memory are likely attributable to the executive function component.

The prefrontal cortex is the primary neural substrate believed to underlie working memory (Funahashi, 2001). Existing evidence links the dopaminergic system to working memory functions and in general, serotonergic manipulations in animals have left working memory operations intact (Robbins, 2005). Therefore, ATD was not expected to directly impair working memory.

The effects of ATD on working memory were explored mostly with two common tasks; backward digit span and the Sternberg Memory Scanning (SMS) task (Table 5). Luciana et al. (2001) found that ATD did not affect forward and backward digits spans, but TRP loading impaired the number of digits recalled during backwards digit span suggesting elevated 5-HT levels restricts information flow leading to working memory impairments. Porter et al. (2003b, 2005) reported inconsistent results for the backward digit span task in healthy elderly, recovered depressed patients, and patients with Alzheimer's disease. The pooled analysis of these two studies revealed no ATD effect on backward digit span (Mace et al., 2008). In a different working memory task, The Paced Auditory Serial Addition Task, participants add each number in a sequence to the preceding number presented. Stewart et al. (2002) found no effect of ATD on performance during this task or during backward digit span in both high and low neuroticism groups.

In the SMS task, participants are presented with a target set of varying size that they must recall on subsequent trials when responding to probes that may or may not contain the target item(s). Two studies employing the SMS task found no effect of ATD in healthy adults or adults with a family history for depression (Harrison et al., 2004; Riedel et al., 1999) while one group reported that ATD slowed overall reaction times (Sobczak et al., 2002). Another assessment of working memory for verbal stimuli is the Nback task which requires participants to respond to a letter probe if it is the same as the letter presented in an earlier trial. Allen et al. (2006) found that ATD did not affect performance on the 2-back version; however, ATD decreased activation in the right superior/ medial frontal gyrus and decreased activation in the posterior cingulate gyrus. Thus ATD does influence the functional activity of regions involved in working memory and executive functions (such as the prefrontal cortex) but the consequences on performance are less evident.

Although the aforementioned ATD studies on short-term and working memory do not converge to give a clear picture of serotonin's influence over these cognitive operations, evidence sways toward the notion that ATD does not affect either short-term memory or working memory in healthy adults.

Spatial working memory tasks require participants to recall the locations of target stimuli and respond on subsequent trials based on the location of the probes presented. The most common spatial working memory task used in ATD studies was the Cambridge Neuropsychological Test Assessment Battery (CANTAB) version where participants search through four, six, or eight boxes for a token without repeating searched boxes (within search error) or through boxes that contained the token on previous trials (between search error). Three ATD studies employing this task reported no treatment effects on any outcome measures (Park et al., 1994; Porter et al., 2003b, 2005) (Table 5). Similarly, no effects of ATD or TRP loading were demonstrated on two other spatial working memory tasks (Harrison et al., 2004; Luciana et al., 2001). Thus converging evidence indicates that ATD does not affect spatial working memory.

Lastly, one task was used to explore the effects of ATD on affective working memory (Table 5). Participants were first presented with target faces, then with a part of a face (eyes, nose or mouth), and responded based on the similarity to target face. ATD did not affect performance. The only effect observed was that TRP loading decreased accuracy for trials with sad faces when the delay between the target and probe was long suggesting impaired maintenance of sad affective content in working memory (Luciana et al., 2001). This solitary finding within the domain of affective working memory is difficult to interpret on its own. In summary, ATD does not appear to affect short-term memory, verbal working memory, spatial working memory or affective working memory.

\subsection{Executive functions}

Executive functions are 'higher order' cognitive operations such as planning, decision-making, anticipation and reasoning that enable individuals to engage in independent and purposeful behavior (Funahashi, 2001; Lezak, 2004). Executive functions allow for control of inhibition, attention, and concept-shifting (Funahashi, 2001). The prefrontal cortex and the dopaminergic system are widely implicated in the control of executive functions but findings evidencing serotonin's contribution to this domain are less robust (Funahashi, 2001; Robbins, 2005). Therefore, ATD is not expected to produce specific deficits in executive functions.

\subsubsection{Planning}

Planning is an executive function involving the identification and organization of the steps and elements necessary to achieve goals (Lezak, 2004). The Tower of London was the most common assessment of planning utilized. Nine ATD studies included the Tower of London assessment and most indicate that ATD does not affect planning (Booij et al., 2005; Hughes et al., 2002, 2003; Murphy et al., 2002; Park et al., 1994; Porter et al., 2005; Schmitt et al., 2000; Sobczak et al., 2002) (Table 6). ATD did not affect the number of excess moves or efficiency of planning in any of these studies although several studies did report some treatment effects. Schmitt et al. (2000) found that ATD decreased decision-making times thereby improving performance in healthy volunteers. Park et al. (1994) reported that ATD improved initiation and execution times in naïve participants but slowed thinking times in participants who were already familiar with the task. Consistent with the former finding, participants showed greater improvement for easier problems during ATD when the task was novel (Hughes et al., 2003). Sobczak et al. (2002) demonstrated that ATD increased response times in participants with a positive family history of bipolar disorder and Kulz et al. (2007) found large effect sizes for decreased number of correct solutions and trends towards increased movement times in patients with OCD. Although some treatment effects on Tower of London performance have been reported, the majority of the findings do not indicate that ATD explicitly affects planning abilities in a consistent manner.

\subsubsection{Decision-making}

Decision-making tasks require responding based on a variety of criteria including probabilistic choice and the delays associated with rewards. In tests of probabilistic choice, participants choose between two outcomes that are associated with rewards or punishments based on their likelihood of occurring. Of the four studies assessing probabilistic choice, two found that ATD improved decision-making based on risks; the group receiving ATD chose the more likely outcome more often or chose an experimental gamble when its associated gains were higher (Rogers et al., 2003; Talbot et al., 2006) (Table 6). It was suggested that ATD may alter the processing of reward cues (Rogers et al., 2003). In contrast, in one study, ATD depreciated the quality of decision-making (Rogers et al., 1999b) and another study found that ATD had no effect on probabilistic choice (Anderson et al., 2003). Similarly, Crean et al. (2002) found that ATD did not affect 
preference for immediate, smaller rewards over larger, delayed rewards. Differences in the outcomes of these studies can be attributed to the use of different tasks and between-subjects designs. Overall, the majority of evidence suggest that ATD does not explicitly affect decision-making.

Two tasks examined the effects of ATD on responding to cuedreinforcements in a Cued-Reinforcement Reaction-Time Task. The task involves identifying an 'odd-one-out' stimulus from an array of stimuli and on occasional trials a cue indicates when a correct response on a subsequent trial will be accompanied by a reward. Cools et al. (2005a) found that ATD impaired the ability to adapt to incentive-motivational cues by abolishing the speeding of responses seen in controls to trials with higher likelihoods of rewards. Another study reported similar findings; however, the abolishing of reward-related speeding after ATD was only seen in participants with a 5-HT transporter genotype associated with vulnerability to affective disorders (Roiser et al., 2006). Taken together, these findings do not provide a clear picture of the effects of ATD on decision-making. The use of between-subjects designs in the majority of these studies and the complexity of the tasks complicates the interpretation of these findings. Given that only two studies reported performance decrements in healthy volunteers or in a vulnerable 5-HT genotype group, two reported no effect and two reported an improvement, ATD does not appear to impair decision-making abilities in healthy adults in a consistent manner.

\subsubsection{Response inhibition}

Response inhibition is an executive function defined as the use of higher-level executive control to suppress an unwanted prepotent response and it is considered to involve input from the prefrontal cortex (Aron and Poldrack, 2005; Funahashi, 2001). Assessments of response inhibition typically involve responding to targets and avoiding responses to distractors (Go/No-Go) or responding to a signal that is occasionally followed by a cue indicating the response must be ceased (Stop Signal Task). All four studies employing traditional Go/No-Go task reported that ATD did not increase error rates in healthy volunteers (Evers et al., 2006b; LeMarquand et al., 1998, 1999; Rubia et al., 2005) (Table 6). However, ATD increased commission errors in participants with a positive family history for alcoholism (LeMarquand et al., 1999). In two fMRI studies, although no cognitive effects during the Go/NoGo task were noted, ATD decreased activation in the dorsomedial prefrontal cortex during performance monitoring (Evers et al., 2006b) and increased activation in the right middle temporal and left middle-superior and inferior temporal gyri (Rubia et al., 2005). Utilizing a different assessment of response inhibition, the Stop Signal Task, two studies reported no effect of ATD (Clark et al., 2005; Cools et al., 2005a) and one found that ATD increased stop RT in participants with a family history of alcoholism and decreased stop RT in the family history negative group (Crean et al., 2002). Overall, the majority of these findings suggest that ATD does not impair response inhibition in healthy adults.

The remainder of the executive function assessments used in ATD studies overlap with the cognitive domain of attention and therefore they will be discussed together.

\subsection{Attention}

Attention is a cognitive domain that is difficult to define however it depends on the reception, selection and filtering of information (Lezak, 2004). Assessments of attention are divided into the following categories: sustained, selective or focused, and divided attention. Attention is mediated by a diffuse network or brain regions including prefrontal, anterior cingulate, and posterior parietal cortices (Kondo et al., 2004; Peelen et al., 2004;
Pollmann, 2004). Several neurotransmitter systems are linked to attentional processes including the cholinergic and dopaminergic systems and 5-HT is implicated in behavioral inhibition (Robbins, 1997, 2005).

\subsubsection{Sustained attention or vigilance}

Sustained attention or vigilance is the capacity to maintain continuous attentional activity or responding over an extended period of time (Lezak, 2004). Letter cancellation tasks tap sustained attention by requiring participants to scan through an array of randomly distributed letters and circle or cross-out a target letter. ATD was not found to affect the number of errors or response times during two types of letter cancellation tasks in healthy volunteers (Luciana et al., 2001; Shansis et al., 2000) (Table 7). However, TRP loading enhanced vigilance evidenced by a decreased the number of omission errors compared to ATD (Luciana et al., 2001).

Several other ATD studies assessing vigilance employed continuous performance tasks (CPT) where participants respond to relatively infrequent events that demand active attention for detection. Three studies reported no effect of ATD on CPT performance in healthy adults or participants classified as highneuroticism individuals (Harrison et al., 2004; Park et al., 1994; Stewart et al., 2002). In contrast, Dougherty et al. (2007) showed that ATD increased commission errors relative to TRP loading and Walderhaug et al. (2002) reported that ATD led to a more lenient response criterion for number targets and caused fewer hits and decreased perceptual sensitivity for shape targets during a modified CPT. Similar findings were later reported by the same group (Walderhaug et al., 2008) but only when the task was novel. The authors suggested that novel situations and 5-HT depletion increase impulsivity and possibly also impair attention.

A different type of CPT is the Vigil task where participants respond only to a single letter target (Vigil K) or only when a specific letter precedes the target letter (Vigil AK). Two studies investigated the effects of ATD with the Vigil task in elderly participants. In one study, ATD increased commission errors during the Vigil AK in healthy elderly (Porter et al., 2003b). The second study tested healthy elderly and recovered depressed patients during the Vigil AK and found that ATD did not affect response latencies or errors (Porter et al., 2005). ATD had no effect on performance in the Vigil task in euthymic bipolar patients (Hughes et al., 2002). In patients with Schizophrenia, ATD did not affect performance on the Vigil A or Vigil AK test overall although commission errors were increased in the first quarter of the test (Golightly et al., 2001). Thus, the majority of the evidence suggest that ATD does not directly alter sustained attention in healthy volunteers, but ATD may impair vigilance in the elderly.

\subsubsection{Selective or focused attention}

Selective or focused attention is the ability to attend preferentially to one or two relevant stimuli while suppressing distractions (Lezak, 2004). A classic assessment of focused attention is the Stroop Colour Word Test where participants must inhibit the tendency to read colour words and instead name the colour of the ink (the Stroop task is also considered to assess response inhibition). Sobczak et al. (2002) and Gallagher et al. (2003) found that ATD did not affect performance during the Stroop task (Table 8). However, in three studies ATD decreased interference scores suggesting that ATD improves focused attention or response inhibition (Booij et al., 2005; Schmitt et al., 2000; Scholes et al., 2007). Of the two fMRI studies, ATD had no effect on performance but increased activation in bilateral mediofrontal cortex, anterior cingulate cortex and left dorsolateral prefrontal cortex (Horacek et al., 2005), regions that are Stroop-task specific (Bench et al., 1993; George et al., 1997; Taylor et al., 1997). In contrast, ATD decreased the interference score and increased 
activity in the anterior cingulate cortex in another fMRI study (Evers et al., 2006a). Evers et al. (2006a) attributed the differences in performance and functional outcomes between these two imaging studies to differences in task requirements and demands. Overall, ATD does not appear to impair response inhibition or focused attention during the Stroop task and may improve these cognitive operations.

Four other studies investigated the effects of ATD on modified Stroop tasks. Hayward et al. (2005) showed no effect of ATD during a Stroop counting task where participants responded according to the number of number words on a screen during interference trials. During another variation of the Stroop counting task which involved counting the number of emotionally loaded words presented, ATD enhanced the emotional interference in healthy adults and recovered depressed patients (Hayward et al., 2005). In an fMRI study, ATD increased the number of errors for negative words but did not affect interference scores or brain activation during an emotional Stroop task (Evers et al., 2006a). Three other variations using emotional stimuli were included in ATD studies. Booij et al. (2005) tested participants' abilities to name the colours of emotionally loaded words and found that ATD increased interference for positively loaded words only. In another study, participants named the colours of emotionally loaded words and smoking-related words in smokers with or without histories of depression (Hitsman et al., 2007). ATD increased interference times for all types of words irrespective of psychiatric history. Lastly, Munafo et al. (2006) examined the effects of ATD on a different emotional Stroop task where participants named the colour of a screen that contained socially threatening or nonsocially threatening words. ATD did not affect colour naming in healthy volunteers or unmedicated patients with histories of depression; however, ATD slowed background colour naming for socially threatening words in medicated patients with histories of depression.

Although the findings of these studies do not provide a clear picture of ATD effects on focused attention it appears that ATD does not impair these cognitive operations and may actually improve them. In addition, the effects of ATD on focused attention may depend on the emotional valence of stimuli.

\subsubsection{Divided attention}

Divided attention is the ability to respond to multiple different tasks simultaneously and thus can be considered the capacity to maintain and shift focused attention according to task demands (Lezak, 2004). The dichotic listening task assesses focused and divided attention in the auditory modality. Participants are presented with different stimuli in each ear and must respond by selectively directing attention according to task prompts. The focused attention subtask requires participants to attend to information presented in one ear while ignoring stimuli presented to the contralateral ear. In the divided attention subtask, participants attend to information presented in both ears simultaneously. Schmitt et al. (2000) reported that ATD improved performance on the focused attention subtask but had no effect on the divided attention subtask (Table 8). In contrast, Sobczak et al. (2002) found no effect of ATD on either subtask. One possible explanation for these discordant outcomes is the use of variable populations; Sobczak et al. (2002) included participants with a wider age range and Schmitt et al. (2000) tested only young participants. These two equivocal studies are likely insufficient to conclude the influence of ATD on divided attention.

\subsubsection{Concept-shifting}

Concept-shifting or set-shifting refers to the cognitive flexibility which allows alternating between types of responding depending on the task conditions and context (Funahashi, 2001). Two versions of a basic test of set-shifting require participants to connect consecutive digits or letters arranged in a circle (Concept-Shifting Task, CST) or randomly distributed on a page (Trail-Making Test, TMT). The first trial(s), where participants connect consecutive numbers (TMT A, CST A) or letters (CST B), are assessments of psychomotor processing speed. In the set-shifting trials, participants alternate between numbers and letters. During these basic tests of set-shifting, ATD had no effect (Gallagher et al., 2003; Hughes et al., 2003; Kulz et al., 2007; Scholtissen et al., 2006; Sobczak et al., 2002) (Table 9).

In the affective Go/No-Go task participants are required to shift between responding to targets that were previously distractors. All four studies using the affective Go/No-Go task found no effect on omission errors (Murphy et al., 2002; Roiser et al., 2007, 2008; Rubinsztein et al., 2001) and three found no effect on response latencies (Roiser et al., 2007, 2008; Rubinsztein et al., 2001). Murphy et al. (2002) showed that ATD increased response times for happy targets only. One study reported that the ATD group did not reduce the number of errors during the easier non-shift trials suggesting ATD caused in an inability to maintain the set possibly due to impaired semantic retrieval (Rubinsztein et al., 2001). In contrast, Roiser et al. (2008) found that ATD attenuated an increase in commissions errors caused by attention to positive distractors. ATD increased BOLD responses to emotionally loaded words compared to neutral words measured with fMRI in the ventral striatum, hippocampus, anterior cingular and dorsolateral prefrontal cortex suggesting ATD alters attentional processing of emotional stimuli by modulating activity in limbic and prefrontal areas (Roiser et al., 2008). Although the findings from the affective Go/No-Go task are somewhat inconsistent, some evidence suggests the effects of ATD depend on the emotional valence of stimuli.

The Wisconsin Card Sorting Task (WCST), a classic conceptshifting assessment, tests the ability to shift response strategies with changes in environmental contingencies based on trial and error feedback. Hughes et al. (2003) and Gallagher et al. (2003) found no affect of ATD on any performance measures during the WCST. However, in Schizophrenics, a trend towards fewer categories completed on the WCST was reported (Golightly et al., 2001).

The intra-dimensional/extra-dimensional (ID/ED) discrimination task, a more complex variation of the WCST, is a more specific method for assessing attentional set-shifting capacities. Five ATD studies using ID/ED task have reported conflicting results. Three studies found no effect of ATD on attentional set-shifting and reversal-learning in healthy adults and euthymic bipolar patients (Hughes et al., 2002, 2003; Talbot et al., 2006). In contrast, Rogers et al. (1999a) found that ATD produced a deficit in reversallearning and Park et al. (1994) showed that ATD caused irrelevant stimuli to distract participants when the task was novel; however, ATD exhibited no effect on the ID and ED shifting trials. Talbot et al. (2006) explained the lack of consistent findings with differences in task demands and intersubject variability in performance masking potential effects.

In a different reversal-learning task where participants selected between stimuli that where advantageous or disadvantageous based on associated rewards or punishments, ATD did not affect errors in reversal-learning in three studies (Evers et al., 2005a,b; Murphy et al., 2002). In the fMRI study, a trend was observed for slower reaction times with ATD and reversal learning was associated with increased activation in the dorsomedial prefrontal cortex, a finding that supports serotonin's capacity to modulate prefrontal activity (Evers et al., 2005a). The reasons for the conflicting findings in the aforementioned studies investigating concept-shifting may include methodological differences such as within- versus between-subjects designs and the use of small 
sample sizes. However, taken together with the findings from other set-shifting tasks, the majority of evidence converge to suggest that ATD does not explicitly affect attentional set-shifting or reversal-learning.

\subsection{Emotional information processing}

A number of ATD studies included tasks that examined responses to emotional stimuli. Although the tasks described below are not exclusively assessments of cognition, their findings are briefly summarized here.

Eight studies investigated the effects of ATD on emotional information processing using facial emotion recognition tasks (Table 10). Participants identified the facial expression of a face and three studies included a variation where participants also categorized the gender of the face. Three studies reported no effect of ATD on the recognition of the emotional expressions in healthy volunteers with or without family histories for depression (Cools et al., 2005b; Fusar-Poli et al., 2007; van der Veen et al., 2007). In contrast, three studies found that ATD decreased recognition accuracies for fearful faces; however, two of these studies included vulnerable populations (Harmer et al., 2003; Marsh et al., 2006; Merens et al., 2008). Merens et al. (2008) showed that ATD decreased fear recognition in remitted depressed patients and Marsh et al. (2006) observed the same effect in participants who were heterozygous carriers of the 5-HT transporter genotype associated with affective disorder vulnerability. Interestingly, in one study, ATD improved recognition of happy facial expressions in healthy volunteers but impaired recognition of happy facial expressions in recovered depressed patients (Hayward et al., 2005). Harmer et al. (2003) also reported that ATD decreased recognition for fearful faces in females and not males, which is consistent with the notion that females are more vulnerable to the cognitive and mood effects of ATD (Booij et al., 2002; Sambeth et al., 2007). Lastly, ATD improved response latencies when identifying facial expressions as neutral or emotional for front-viewed faces but not for profiles (Williams et al., 2007). The authors suggested that the serotonergic system is more involved in the recognition of faces directed at the observer and that ATD improved response times through diminished recruitment of higher cortical function.

Four of these studies included neuroimaging and in all four ATD affected brain activation in regions involved in emotional processing such as the amygdala, hippocampus, cingulate and prefrontal cortices (Cools et al., 2005b; Fusar-Poli et al., 2007; van der Veen et al., 2007; Williams et al., 2007). Thus, the effects of ATD extend to cognitive operations involved in the processing of emotional information and diminished 5-HT in anxiety disorders may contribute to the enhanced sensitivity to threat-related stimuli (Cools et al., 2005b).

In summary, some evidence suggest that ATD negatively affects the recognition of fearful faces and this effect may be mediated by brain regions involved in emotional processing.

\section{Conclusions and new insights}

In this paper, we reviewed the effect of ATD on specific cognitive processes. Based on current evidence, ATD impairs consolidation of episodic memory for verbal information in the visual domain and potentially also non-verbal learning although most non-verbal learning studies have produced negative findings. This profile of impairments suggests that the serotonergic system contributes to episodic memory functioning, likely through the serotonergic projection to the hippocampus, a neural substrate known to underlie certain memory operations including episodic memory (Ferbinteanu et al., 2006). Given that verbal skills were a late acquisition in human evolution, it is possible that the neural systems underlying specifically verbal memory are more sensitive to disruptions in serotonergic activity, although this explanation is somewhat speculative, it is testable as a potential new insight.

ATD consistently leaves semantic memory intact, suggesting that serotonergic activity does not influence the retrieval component of declarative memory. However, few tasks examined the effects of ATD on semantic memory and the inclusion of a larger variety of tasks is necessary to substantiate existing findings. With respect to short-term and working memory, the majority of studies converge to support the notion that 5-HT depletion does not influence short-term memory or verbal, spatial and affective, working memory.

Attentional processes seem to be unimpaired by ATD and some findings even suggest that ATD improves focused attention. In terms of executive functions, ATD does not appear to impair planning, response inhibition, or decision-making. Lastly, the majority of findings suggest that psychomotor processing is unaffected by ATD.

Episodic memory is known to depend heavily on the hippocampus and temporal lobes (Ferbinteanu et al., 2006). The effects of ATD on episodic memory consolidation suggest that the serotonergic projection to the hippocampus contributes to episodic memory functioning. In contrast, attention is mediated by a more diverse network of neural substrates including the prefrontal, anterior cingulate, and posterior parietal cortices (Kondo et al., 2004; Peelen et al., 2004; Pollmann, 2004). In addition, the prefrontal cortex, a crucial substrate of attention, working memory and executive functions, is innervated by multiple neurotransmitter systems and depleting solely 5-HT may be insufficient to compromise prefrontal functioning (Robbins, 1997; Robbins, 2005). Since ATD does not robustly impact attentional processes, working memory, or executive functions, the serotonergic system may be less critically involved in the functioning of these cognitive domains. Lastly, given that attentional processes are unaffected or may improve after ATD, the effects of ATD on episodic memory are likely independent of the effects of ATD on attentional processes.

One interesting finding is that ATD consistently impaired episodic memory but not spatial memory, two cognitive domains known to depend on the hippocampus (Burgess et al., 2002). A variety of neurotransmitter systems influence hippocampal activity (Vizi and Kiss, 1998) and animal research has provided some insight into the underlying neuroanatomical and neurochemical processes occurring in the hippocampus that mediate different cognitive and behavioral functions. Steckler et al. (1998a) described two neural networks mediating spatial and non-spatial recognition memory in rats, however, the role of individual neurotransmitter systems in these processes was less evident (Steckler et al., 1998b). Another group found that although serotonin and acetylcholine release in the hippocampus occurred during performance of a spatial memory task in rats, acetylcholine release was implicated in spatial memory operations while serotonin was believed to be involved in the reward-related feeding behaviors (Stancampiano et al., 1999). The extent that these findings generalize to humans is unclear, however, some evidence from animal studies suggest that the different neural networks and possibly different neurotransmitter systems contribute to hippocampal spatial and episodic memory functioning and this may explain the differential effects of ATD on spatial and episodic memory. Perhaps episodic memory is more dependent on serotonergic activity than spatial memory.

Females experience greater episodic memory impairments compared to males during verbal learning following ATD. The most convincing evidence for this assertion comes from a mega-analysis performed by Sambeth et al. (2007) which found that the effects of 
ATD on immediate recall in verbal learning tasks were more pronounced in females. This result parallels the notion that females are more vulnerable to the mood effects of ATD (Booij et al., 2002; Jans et al., 2007). It is unclear why females appear to be more vulnerable to both the cognitive and mood effects of ATD, however a common underlying reason may explain ATD effects on both cognitive and mood. Booij et al. (2002) offered a number of potential explanations for the increased serotonergic vulnerability to ATD effects on mood in females including gender differences in central and peripheral 5-HT metabolism and hormonal factors. However, whether these are valid explanations for ATD mood effects in females and whether serotonergic vulnerability applies to the cognitive effects of ATD in females remains elusive.

In the majority of cases, ATD effects are equivalent for psychiatric populations and healthy volunteers. However, some evidence indicates ATD impacts vulnerable populations more profoundly than healthy individuals particularly for episodic memory, emotional information processing, sustained attention and some executive functions including planning and decisionmaking. The effects of ATD on episodic memory appear to be more pronounced in healthy elderly, depressed patients, and individuals with the vulnerable serotonin transporter genotype. Emotional information processing in patients with histories for depression and vulnerable serotonin transporter genotypes may also be affected more by ATD. Other isolated findings included impaired vigilance and verbal fluency in healthy elderly, decision-making deficits in the vulnerable serotonin transporter genotype, impaired planning in patients with family histories for bipolar disorders and OCD patients, and response inhibition deficits in patients with family histories for alcoholism. The reasons underlying the differential effects of ATD on certain cognitive domains in certain psychiatric populations are unclear. However, serotonergic vulnerability and possibly mood effects may contribute to the disparities in findings between healthy participants and vulnerable groups, although these are speculative explanations and further research is necessary to clarify these discrepancies between healthy and psychiatric populations. It is noteworthy that the cognitive effects of ATD in healthy participants are independent of mood changes.

ATD has been achieved using various methodologies, such as amino acid mixtures of varying doses and gelatin-based protein drinks. Variations in methodologies and in particular amino acid doses raise the issue of whether these differences in manipulation modulate the cognitive outcomes. If so, inadequate depletion in some cases may have led to false negative results. An examination of Table 1 which describes the types of manipulations used and the amount of depletion established in the studies presented in this review reveals that the type of manipulation does not affect depletion substantially. TRP ratio changes in the six studies using the lowest dose of an amino acid drink (25-32 g) were on average as high as $72 \%$. On the other hand, the ratio changes achieved when using 100-105 g of an amino acid mixture (the majority of studies, 42 in total) were on average $79 \%$. Studies using intermediate dosages (50-86 g, a total of 28 studies) revealed an average depletion of $75 \%$. Since, on average, all studies show similar depletion scores, negative outcomes were likely not due to inadequate depletion.

Researching the effects of serotonergic manipulations is complicated by many factors that were described earlier. Pooled analyses of existing studies have provided substantial clarification of the cognitive effects of ATD in some domains. Repeatedmeasures designs are beneficial because they have more power to detect effects and require fewer participants than betweensubjects designs, however, order effects are difficult to interpret and certain types of tasks require between-subjects designs to avoid learning and practice effects. Future studies should attempt to investigate the effects of ATD in larger samples on a variety of tasks that span across multiple cognitive domains to elucidate the role of the serotonergic system within each cognitive domain.

As mentioned earlier, serotonergic dysfunction accompanies a variety of psychiatric illnesses including depression (Arango et al., 2002; Delgado, 2000) and anxiety disorders (Deakin, 1998; Stein and Stahl, 2000) and these conditions often present with cognitive impairments that may be related to serotonergic dysfunction (Bearden et al., 2001; Elvevag and Goldberg, 2000; Porter et al., 2003a). Existing evidence from ATD studies substantiates the notion that diminished 5-HT levels are contributing to the memory impairment in these conditions. The search for efficacious pharmacotherapies should continue to explore the prospect of serotonergic agents in treating specifically the memory symptoms associated with the various psychiatric illnesses with underlying serotonergic etiologies.

\section{References}

Abbott, F.V., Etienne, P., Franklin, K.B., Morgan, M.J., Sewitch, M.J., Young, S.N., 1992. Acute tryptophan depletion blocks morphine analgesia in the cold-pressor test in humans. Psychopharmacology (Berl) 108 (1-2), 60-66.

Ahveninen, J., Jaaskelainen, I.P., Pennanen, S., Liesivuori, J., Ilmoniemi, R.J., Kahkonen, S., 2003. Auditory selective attention modulated by tryptophan depletion in humans. Neurosci. Lett. 340 (3), 181-184.

Ahveninen, J., Kahkonen, S., Pennanen, S., Liesivuori, J., Ilmoniemi, R.J., Jaaskelainen, I.P., 2002. Tryptophan depletion effects on EEG and MEG responses suggest serotonergic modulation of auditory involuntary attention in humans. Neuroimage 16 (4), 1052-1061.

Allen, P.P., Cleare, A.J., Lee, F., Fusar-Poli, P., Tunstall, N., Fu, C.H., Brammer, M.J., McGuire, P.K., 2006. Effect of acute tryptophan depletion on pre-frontal engagement. Psychopharmacology (Berl) 187 (4), 486-497.

Amin, Z., Gueorguieva, R., Cappiello, A., Czarkowski, K.A., Stiklus, S., Anderson, G.M., Naftolin, F., Epperson, C.N., 2006. Estradiol and tryptophan depletion interact to modulate cognition in menopausal women. Neuropsychopharmacology 31 (11), 2489-2497.

Anderson, I.M., Richell, R.A., Bradshaw, C.M., 2003. The effect of acute tryptophan depletion on probabilistic choice. J. Psychopharmacol. 17 (1), 3-7.

Arango, V., Underwood, M.D., Mann, J.J., 2002. Serotonin brain circuits involved in major depression and suicide. Prog. Brain Res. 136, 443-453.

Aron, A.R., Poldrack, R.A., 2005. The cognitive neuroscience of response inhibition: relevance for genetic research in attention-deficit/hyperactivity disorder. Biol. Psychiatry 57 (11), 1285-1292.

Azmitia, E.C., 1978. The serotonin-producing neurons of the midbrain and dosral nuclei. In: Handbook of Psychopharmacology, Plenum Press, New York.

Baddeley, A., 1998. Working memory. C. R. Acad. Sci. III 321 (2-3), 167-173.

Barnes, C.A., 1988. Spatial learning and memory processes: the search for their neurobiological mechanisms in the rat. Trends Neurosci. 11 (4), 163-169.

Barnes, N.M., Sharp, T., 1999. A review of central 5-HT receptors and their function. Neuropharmacology 38 (8), 1083-1152.

Bearden, C.E., Hoffman, K.M., Cannon, T.D., 2001. The neuropsychology and neuroanatomy of bipolar affective disorder: a critical review. Bipolar Disord. 3 (3), $106-150$.

Bench, C.J., Frith, C.D., Grasby, P.M., Friston, K.J., Paulesu, E., Frackowiak, R.S., Dolan, R.J., 1993. Investigations of the functional anatomy of attention using the Stroop test. Neuropsychologia 31 (9), 907-922.

Biggio, G., Fadda, F., Fanni, P., Tagliamonte, A., Gessa, G.L., 1974. Rapid depletion of serum tryptophan, brain tryptophan, serotonin and 5-hydroxyindoleacetic acid by a tryptophan-free diet. Life Sci. 14 (7), 1321-1329.

Blokland, A., Lieben, C., Deutz, N.E., 2002. Anxiogenic and depressive-like effects, but no cognitive deficits, after repeated moderate tryptophan depletion in the rat. J. Psychopharmacol. 16 (1), 39-49.

Boadle-Biber, M.C., 1993. Regulation of serotonin synthesis. Prog. Biophys. Mol. Biol. 60 (1), 1-15.

Booij, L., Van der Does, A.J., Haffmans, P.M., Riedel, W.J., Fekkes, D., Blom, M.J., 2005. The effects of high-dose and low-dose tryptophan depletion on mood and cognitive functions of remitted depressed patients. J. Psychopharmacol. 19 (3), 267-275.

Booij, L., Van der Does, A.J., Riedel, W.J., 2003. Monoamine depletion in psychiatric and healthy populations: review. Mol. Psychiatry 8 (12), 951-973.

Booij, L., Van der Does, W., Benkelfat, C., Bremner, J.D., Cowen, P.J., Fava, M., Gillin, C., Leyton, M., Moore, P., Smith, K.A., Van der Kloot, W.A., 2002. Predictors of mood response to acute tryptophan depletion. A reanalysis. Neuropsychopharmacology 27 (5), 852-861.

Buhot, M.C., 1997. Serotonin receptors in cognitive behaviors. Curr. Opin. Neurobiol. 7 (2), 243-254.

Burgess, N., Maguire, E.A., O'Keefe, J., 2002. The human hippocampus and spatial and episodic memory. Neuron 35 (4), 625-641.

Burgund, E.D., Marsolek, C.J., Luciana, M., 2003. Serotonin levels influence patterns of repetition priming. Neuropsychology 17 (1), 161-170. 
Clark, L., Roiser, J.P., Cools, R., Rubinsztein, D.C., Sahakian, B.J., Robbins, T.W., 2005. Stop signal response inhibition is not modulated by tryptophan depletion or the serotonin transporter polymorphism in healthy volunteers: implications for the 5-HT theory of impulsivity. Psychopharmacology (Berl) 182 (4), 570-578.

Cools, R., Blackwell, A., Clark, L., Menzies, L., Cox, S., Robbins, T.W., 2005a. Tryptophan depletion disrupts the motivational guidance of goal-directed behavior as a function of trait impulsivity. Neuropsychopharmacology 30 (7), 1362-1373.

Cools, R., Calder, A.J., Lawrence, A.D., Clark, L., Bullmore, E., Robbins, T.W., 2005b. Individual differences in threat sensitivity predict serotonergic modulation of amygdala response to fearful faces. Psychopharmacology (Berl) 180 (4), 670679.

Cowan, N., 2008. Chapter 20 What are the differences between long-term, shortterm, and working memory? Prog. Brain Res. 169, 323-338.

Crean, J., Richards, J.B., de Wit, H., 2002. Effect of tryptophan depletion on impulsive behavior in men with or without a family history of alcoholism. Behav. Brain Res. 136 (2), 349-357.

Deakin, J.F., 1998. The role of serotonin in panic, anxiety and depression. Int. Clin. Psychopharmacol. 13 (Suppl. 4), S1-S5.

Delgado, P.L., 2000. Depression: the case for a monoamine deficiency. J. Clin. Psychiatry 61 (Suppl. 6), 7-11.

Dougherty, D.M., Marsh, D.M., Mathias, C.W., Dawes, M.A., Bradley, D.M., Morgan, C.J., Badawy, A.A., 2007. The effects of alcohol on laboratory-measured impulsivity after L: tryptophan depletion or loading. Psychopharmacology (Berl) 193 (1), 137-150.

Ellenbogen, M.A., Young, S.N., Dean, P., Palmour, R.M., Benkelfat, C., 1996. Mood response to acute tryptophan depletion in healthy volunteers: sex differences and temporal stability. Neuropsychopharmacology 15 (5), 465-474.

Elvevag, B., Goldberg, T.E., 2000. Cognitive impairment in schizophrenia is the core of the disorder. Crit. Rev. Neurobiol. 14 (1), 1-21.

Epperson, C.N., Amin, Z., Naftolin, F., Cappiello, A., Czarkowski, K.A., Stiklus, S., Anderson, G.M., Krystal, J.H., 2007. The resistance to depressive relapse in menopausal women undergoing tryptophan depletion: preliminary findings. J. Psychopharmacol. 21 (4), 414-420.

Evers, E.A., Cools, R., Clark, L., van der Veen, F.M., Jolles, J., Sahakian, B.J., Robbins, T.W., 2005a. Serotonergic modulation of prefrontal cortex during negative feedback in probabilistic reversal learning. Neuropsychopharmacology 30 (6), 1138-1147.

Evers, E.A., Tillie, D.E., van der Veen, F.M., Lieben, C.K., Jolles, J., Deutz, N.E., Schmitt, J.A., 2005b. Effects of a novel method of acute tryptophan depletion on plasma tryptophan and cognitive performance in healthy volunteers. Psychopharmacology (Berl) 178 (1), 92-99.

Evers, E.A., van der Veen, F.M., Fekkes, D., Jolles, J., 2007. Serotonin and cognitive flexibility: neuroimaging studies into the effect of acute tryptophan depletion in healthy volunteers. Curr. Med. Chem. 14 (28), 2989-2995.

Evers, E.A., van der Veen, F.M., Jolles, J., Deutz, N.E., Schmitt, J.A., 2006a. Acute tryptophan depletion improves performance and modulates the BOLD response during a Stroop task in healthy females. Neuroimage 32 (1), 248-255.

Evers, E.A., van der Veen, F.M., van Deursen, J.A., Schmitt, J.A., Deutz, N.E., Jolles, J., 2006b. The effect of acute tryptophan depletion on the BOLD response during performance monitoring and response inhibition in healthy male volunteers. Psychopharmacology (Berl) 187 (2), 200-208.

Ferbinteanu, J., Kennedy, P.J., Shapiro, M.L., 2006. Episodic memory-from brain to mind. Hippocampus 16 (9), 691-703.

Fernstrom, J.D., 1979. Diet-induced changes in plasma amino acid pattern: effects on the brain uptake of large neutral amino acids, and on brain serotonin synthesis. J. Neural. Transm. Suppl. (15), 55-67.

Fernstrom, J.D., Faller, D.V., 1978. Neutral amino acids in the brain: changes in response to food ingestion. J. Neurochem. 30 (6), 1531-1538.

Funahashi, S., 2001. Neuronal mechanisms of executive control by the prefrontal cortex. Neurosci. Res. 39 (2), 147-165.

Fusar-Poli, P., Allen, P., Lee, F., Surguladze, S., Tunstall, N., Fu, C.H., Brammer, M.J., Cleare, A.J., McGuire, P.K., 2007. Modulation of neural response to happy and sad faces by acute tryptophan depletion. Psychopharmacology (Berl) 193 (1), 31-44.

Fusar-Poli, P., Allen, P., McGuire, P., Placentino, A., Cortesi, M., Perez, J., 2006. Neuroimaging and electrophysiological studies of the effects of acute tryptophan depletion: a systematic review of the literature. Psychopharmacology (Berl) 188 (2), 131-143.

Gallagher, P., Massey, A.E., Young, A.H., McAllister-Williams, R.H., 2003. Effects of acute tryptophan depletion on executive function in healthy male volunteers. BMC Psychiatry 3, 10.

George, M.S., Ketter, T.A., Parekh, P.I., Rosinsky, N., Ring, H.A., Pazzaglia, P.J., Marangell, L.B., Callahan, A.M., Post, R.M., 1997. Blunted left cingulate activation in mood disorder subjects during a response interference task (the Stroop). J. Neuropsychiatry Clin. Neurosci. 9 (1), 55-63.

Golightly, K.L., Lloyd, J.A., Hobson, J.E., Gallagher, P., Mercer, G., Young, A.H., 2001. Acute tryptophan depletion in schizophrenia. Psychol. Med. 31 (1), 75-84.

Harmer, C.J., Rogers, R.D., Tunbridge, E., Cowen, P.J., Goodwin, G.M., 2003. Tryptophan depletion decreases the recognition of fear in female volunteers. Psychopharmacology (Berl) 167 (4), 411-417.

Harrison, B.J., Olver, J.S., Norman, T.R., Burrows, G.D., Wesnes, K.A., Nathan, P.J., 2004. Selective effects of acute serotonin and catecholamine depletion on memory in healthy women. J. Psychopharmacol. 18 (1), 32-40.

Harrison, B.J., Olver, J.S., Norman, T.R., Nathan, P.J., 2002. Brain monoamines and early visual information-processing speed. Int. J. Neuropsychopharmacol. 5 (4), 295-300.
Hayward, G., Goodwin, G.M., Cowen, P.J., Harmer, C.J., 2005. Low-dose tryptophan depletion in recovered depressed patients induces changes in cognitive processing without depressive symptoms. Biol. Psychiatry 57 (5), 517-524.

Hitsman, B., Spring, B., Pingitore, R., Munafo, M., Hedeker, D., 2007. Effect of tryptophan depletion on the attentional salience of smoking cues. Psychopharmacology (Berl) 192 (3), 317-324.

Horacek, J., Zavesicka, L., Tintera, J., Dockery, C., Platilova, V., Kopecek, M., Spaniel, F., Bubenikova, V., Hoschl, C., 2005. The effect of tryptophan depletion on brain activation measured by functional magnetic resonance imaging during the Stroop test in healthy subjects. Physiol. Res. 54 (2), 235-244.

Hughes, J.H., Gallagher, P., Stewart, M.E., Matthews, D., Kelly, T.P., Young, A.H., 2003 The effects of acute tryptophan depletion on neuropsychological function. J. Psychopharmacol. 17 (3), 300-309.

Hughes, J.H., Gallagher, P., Young, A.H., 2002. Effects of acute tryptophan depletion on cognitive function in euthymic bipolar patients. Eur. Neuropsychopharmacol. 12 (2), 123-128.

Jacobs, B.L., Azmitia, E.C., 1992. Structure and function of the brain serotonin system. Physiol. Rev. 72 (1), 165-229.

Jans, L.A., Riedel, W.J., Markus, C.R., Blokland, A., 2007. Serotonergic vulnerability and depression: assumptions, experimental evidence and implications. Mol. Psychiatry 12 (6), 522-543.

Kahkonen, S., Jaaskelainen, I.P., Pennanen, S., Liesivuori, J., Ahveninen, J., 2002. Acute tryptophan depletion decreases intensity dependence of auditory evoked magnetic N1/P2 dipole source activity. Psychopharmacology (Berl) 164 (2), 221 227.

Kent, J.M., Coplan, J.D., Gorman, J.M., 1998. Clinical utility of the selective serotonin reuptake inhibitors in the spectrum of anxiety. Biol. Psychiatry 44 (9), 812-824.

Kilkens, T.O., Honig, A., van Nieuwenhoven, M.A., Riedel, W.J., Brummer, R.J., 2004 Acute tryptophan depletion affects brain-gut responses in irritable bowel syndrome patients and controls. Gut 53 (12), 1794-1800.

Klaassen, T., Riedel, W.J., Deutz, N.E., Van Praag, H.M., 2002. Mood congruent memory bias induced by tryptophan depletion. Psychol. Med. 32 (1), 167-172

Klaassen, T., Riedel, W.J., Deutz, N.E., van Someren, A., van Praag, H.M., 1999. Specificity of the tryptophan depletion method. Psychopharmacology (Berl) 141 (3), 279-286.

Kondo, H., Osaka, N., Osaka, M., 2004. Cooperation of the anterior cingulate cortex and dorsolateral prefrontal cortex for attention shifting. Neuroimage 23 (2) 670-679.

Kulz, A.K., Meinzer, S., Kopasz, M., Voderholzer, U., 2007. Effects of tryptophan depletion on cognitive functioning, obsessive-compulsive symptoms and mood in obsessive-compulsive disorder: preliminary results. Neuropsychobiology 56 (2-3), 127-131.

Lee, M.A., Meltzer, H.Y., 2001. 5-HT(1A) receptor dysfunction in female patients with schizophrenia. Biol. Psychiatry 50 (10), 758-766.

LeMarquand, D.G., Benkelfat, C., Pihl, R.O., Palmour, R.M., Young, S.N., 1999. Behavioral disinhibition induced by tryptophan depletion in nonalcoholic young men with multigenerational family histories of paternal alcoholism. Am. J. Psychiatry 156 (11), 1771-1779.

LeMarquand, D.G., Pihl, R.O., Young, S.N., Tremblay, R.E., Seguin, J.R., Palmour, R.M., Benkelfat, C., 1998. Tryptophan depletion, executive functions, and disinhibition in aggressive, adolescent males. Neuropsychopharmacology 19 (4), 333-341.

Lezak, M.D., 2004. Neuropsychological Assessment. Oxford University Press, New York.

Lieben, C.K., Blokland, A., Westerink, B., Deutz, N.E., 2004. Acute tryptophan and serotonin depletion using an optimized tryptophan-free protein-carbohydrate mixture in the adult rat. Neurochem. Int. 44 (1), 9-16.

Luciana, M., Burgund, E.D., Berman, M., Hanson, K.L., 2001. Effects of tryptophan loading on verbal, spatial and affective working memory functions in healthy adults. J. Psychopharmacol. 15 (4), 219-230.

Mace, J., Porter, R., O'Brien, J., Gallagher, P., 2008. Cognitive effects of acute tryptophan depletion in the healthy elderly. Acta Neuropsychiatrica 20, 78-86.

Mace, S., Taylor, D., 2000. Selective serotonin reuptake inhibitors: a review of efficacy and tolerability in depression. Expert. Opin. Pharmacother. 1 (5), 917-933.

Mahmood, T., Silverstone, T., 2001. Serotonin and bipolar disorder. J. Affect Disord. 66 (1), 1-11.

Marsh, A.A., Finger, E.C., Buzas, B., Soliman, N., Richell, R.A. Vythilingham, M., Pine, D.S., Goldman, D., Blair, R.J., 2006. Impaired recognition of fear facial expressions in 5-HTTLPR S-polymorphism carriers following tryptophan depletion. Psychopharmacology (Berl) 189 (3), 387-394.

McAllister-Williams, R.H., Massey, A.E., Rugg, M.D., 2002. Effects of tryptophan depletion on brain potential correlates of episodic memory retrieval. Psychopharmacology (Berl) 160 (4), 434-442.

Merens, W., Booij, L., Haffmans, P.M., Van der Does, A.J., 2008. The effects of experimentally lowered serotonin function on emotional information processing and memory in remitted depressed patients. J. Psychopharmacol. 22 (6), 653-662.

Morris, J.S., Smith, K.A., Cowen, P.J., Friston, K.J., Dolan, R.J., 1999. Covariation of activity in habenula and dorsal raphe nuclei following tryptophan depletion. Neuroimage $10(2), 163-172$.

Munafo, M.R., Hayward, G., Harmer, C., 2006. Selective processing of social threat cues following acute tryptophan depletion. J. Psychopharmacol. 20 (1), 33-39.

Murphy, F.C., Smith, K.A., Cowen, P.J., Robbins, T.W., Sahakian, B.J., 2002. The effects of tryptophan depletion on cognitive and affective processing in healthy volunteers. Psychopharmacology (Berl) 163 (1), 42-53. 
Nieuwenhuys, R., 1985. Chemoarchitecture of the Brain. Springer-Verlag, Berlin, New York.

Nishizawa, S., Benkelfat, C., Young, S.N., Leyton, M., Mzengeza, S., de Montigny, C., Blier, P., Diksic, M., 1997. Differences between males and females in rates of serotonin synthesis in human brain. Proc. Natl. Acad. Sci. USA 94 (10), 53085313.

Oldendorf, W.H., Szabo, J., 1976. Amino acid assignment to one of three blood-brain barrier amino acid carriers. Am. J. Physiol. 230 (1), 94-98.

Oldman, A.D., Walsh, A.E.S., Salkovskis, P., Laver, D.A., Cowen, P.J., 1994. Effect of acute tryptophan depletion on mood and appetite in healthy female volunteers. J. Psychopharmacol. 8 (1), 8-13.

Pakarinen, S., Takegata, R., Rinne, T., Huotilainen, M., Naatanen, R., 2007. Measurement of extensive auditory discrimination profiles using the mismatch negativity (MMN) of the auditory event-related potential (ERP). Clin. Neurophysiol 118 (1), 177-185.

Park, S.B., Coull, J.T., McShane, R.H., Young, A.H., Sahakian, B.J., Robbins, T.W., Cowen, P.J., 1994. Tryptophan depletion in normal volunteers produces selective impairments in learning and memory. Neuropharmacology 33 (3-4), 575-588.

Peelen, M.V., Heslenfeld, D.J., Theeuwes, J., 2004. Endogenous and exogenous attention shifts are mediated by the same large-scale neural network. Neuroimage 22 (2), 822-830.

Pollmann, S., 2004. Anterior prefrontal cortex contributions to attention control Exp. Psychol. 51 (4), 270-278.

Porter, R.J., Gallagher, P., Thompson, J.M., Young, A.H., 2003a. Neurocognitive impairment in drug-free patients with major depressive disorder. Br. J. Psychiatry $182,214-220$.

Porter, R.J., Lunn, B.S., O’Brien, J.T., 2003b. Effects of acute tryptophan depletion on cognitive function in Alzheimer's disease and in the healthy elderly. Psychol. Med. 33 (1), 41-49.

Porter, R.J., Lunn, B.S., Walker, L.L., Gray, J.M., Ballard, C.G., O’Brien, J.T., 2000. Cognitive deficit induced by acute tryptophan depletion in patients with Alzheimer's disease. Am. J. Psychiatry 157 (4), 638-640.

Porter, R.J., Phipps, A.J., Gallagher, P., Scott, A., Stevenson, P.S., O’Brien, J.T., 2005 Effects of acute tryptophan depletion on mood and cognitive functioning in older recovered depressed subjects. Am. J. Geriatr. Psychiatry 13 (7), 607-615.

Reilly, J.G., McTavish, S.F., Young, A.H., 1997. Rapid depletion of plasma tryptophan: a review of studies and experimental methodology. J. Psychopharmacol. 11 (4) 381-392.

Riedel, W.J., 2004. Cognitive changes after acute tryptophan depletion: what can they tell us? Psychol. Med. 34 (1), 3-8.

Riedel, W.J., Klaassen, T., Deutz, N.E., van Someren, A., van Praag, H.M., 1999. Tryptophan depletion in normal volunteers produces selective impairment in memory consolidation. Psychopharmacology (Berl) 141 (4), 362-369.

Riedel, W.J., Klaassen, T., Schmitt, J.A., 2002. Tryptophan, mood, and cognitive function. Brain Behav. Immun. 16 (5), 581-589.

Riedel, W.J., Sobczak, S., Schmitt, J.A., 2003. Tryptophan modulation and cognition. Adv. Exp. Med. Biol. 527, 207-213.

Robbins, T.W., 1997. Arousal systems and attentional processes. Biol. Psychol. 45 $(1-3), 57-71$

Robbins, T.W., 2005. Chemistry of the mind: neurochemical modulation of prefrontal cortical function. J. Comp. Neurol. 493 (1), 140-146.

Rogers, R.D., Blackshaw, A.J., Middleton, H.C., Matthews, K., Hawtin, K., Crowley, C., Hopwood, A., Wallace, C., Deakin, J.F., Sahakian, B.J., Robbins, T.W., 1999a. Tryptophan depletion impairs stimulus-reward learning while methylphenidate disrupts attentional control in healthy young adults: implications for the monoaminergic basis of impulsive behaviour. Psychopharmacology (Berl) 146 (4), 482-491.

Rogers, R.D., Everitt, B.J., Baldacchino, A., Blackshaw, A.J., Swainson, R., Wynne, K., Baker, N.B., Hunter, J., Carthy, T., Booker, E., London, M., Deakin, J.F., Sahakian, B.J., Robbins, T.W., 1999b. Dissociable deficits in the decision-making cognition of chronic amphetamine abusers, opiate abusers, patients with focal damage to prefrontal cortex, and tryptophan-depleted normal volunteers: evidence for monoaminergic mechanisms. Neuropsychopharmacology 20 (4), 322-339.

Rogers, R.D., Tunbridge, E.M., Bhagwagar, Z., Drevets, W.C., Sahakian, B.J., Carter, C.S., 2003. Tryptophan depletion alters the decision-making of healthy volunteers through altered processing of reward cues. Neuropsychopharmacology 28 (1), 153-162.

Roiser, J.P., Blackwell, A.D., Cools, R., Clark, L., Rubinsztein, D.C., Robbins, T.W. Sahakian, B.J., 2006. Serotonin transporter polymorphism mediates vulnerability to loss of incentive motivation following acute tryptophan depletion. Neuropsychopharmacology 31 (10), 2264-2272.

Roiser, J.P., Levy, J., Fromm, S.J., Wang, H., Hasler, G., Sahakian, B.J., Drevets, W.C., 2008. The effect of acute tryptophan depletion on the neural correlates of emotional processing in healthy volunteers. Neuropsychopharmacology 33 (8), 1992-2006.

Roiser, J.P., Muller, U., Clark, L., Sahakian, B.J., 2007. The effects of acute tryptophan depletion and serotonin transporter polymorphism on emotional processing in memory and attention. Int. J. Neuropsychopharmacol. 10 (4), 449-461.

Rubia, K., Lee, F., Cleare, A.J., Tunstall, N., Fu, C.H., Brammer, M., McGuire, P., 2005. Tryptophan depletion reduces right inferior prefrontal activation during response inhibition in fast, event-related fMRI. Psychopharmacology (Berl) 179 (4), 791-803.

Rubinsztein, J.S., Rogers, R.D., Riedel, W.J., Mehta, M.A., Robbins, T.W., Sahakian, B.J., 2001. Acute dietary tryptophan depletion impairs maintenance of "affective set" and delayed visual recognition in healthy volunteers. Psychopharmacology (Berl) 154 (3), 319-326.
Ruhe, H.G., Mason, N.S., Schene, A.H., 2007. Mood is indirectly related to serotonin, norepinephrine and dopamine levels in humans: a meta-analysis of monoamine depletion studies. Mol. Psychiatry 12 (4), 331-359.

Sambeth, A., Blokland, A., Harmer, C.J., Kilkens, T.O., Nathan, P.J., Porter, R.J., Schmitt, J.A., Scholtissen, B., Sobczak, S., Young, A.H., Riedel, W.J., 2007. Sex differences in the effect of acute tryptophan depletion on declarative episodic memory: a pooled analysis of nine studies. Neurosci. Biobehav. Rev. 31 (4), 516-529.

Sambeth, A., Riedel, W.J., Tillie, D.E., Blokland, A., Postma, A., Schmitt, J.A., 2009 Memory impairments in humans after acute tryptophan depletion using a novel gelatin-based protein drink. J. Psychopharmacol. 23, 56-64.

Schmitt, J.A., Jorissen, B.L., Sobczak, S., van Boxtel, M.P., Hogervorst, E., Deutz, N.E., Riedel, W.J., 2000. Tryptophan depletion impairs memory consolidation but improves focussed attention in healthy young volunteers. J. Psychopharmacol. 14 (1), 21-29.

Schmitt, J.A., Wingen, M., Ramaekers, J.G., Evers, E.A., Riedel, W.J., 2006. Serotonin and human cognitive performance. Curr. Pharm. Des. 12 (20), 2473-2486.

Scholes, K.E., Harrison, B.J., O’Neill, B.V., Leung, S., Croft, R.J., Pipingas, A., Phan, K.L., Nathan, P.J., 2007. Acute serotonin and dopamine depletion improves attentional control: findings from the Stroop task. Neuropsychopharmacology 32 (7), $1600-1610$.

Scholtissen, B., Verhey, F.R., Adam, J.J., Prickaerts, J., Leentjens, A.F., 2006. Effects of acute tryptophan depletion on cognition, memory and motor performance in Parkinson's disease. J. Neurol. Sci. 248 (1-2), 259-265.

Shansis, F.M., Busnello, J.V., Quevedo, J., Forster, L., Young, S., Izquierdo, I., Kapczinski, F., 2000. Behavioural effects of acute tryptophan depletion in healthy male volunteers. J. Psychopharmacol. 14 (2), 157-163.

Smith, K.A., Morris, J.S., Friston, K.J., Cowen, P.J., Dolan, R.J., 1999. Brain mechanisms associated with depressive relapse and associated cognitive impairment following acute tryptophan depletion. Br. J. Psychiatry 174, 525-529.

Smith, S.E., Pihl, R.O., Young, S.N., Ervin, F.R., 1987. A test of possible cognitive and environmental influences on the mood lowering effect of tryptophan depletion in normal males. Psychopharmacology (Berl) 91 (4), 451-457.

Sobczak, S., Riedel, W.J., Booij, I., Aan Het Rot, M., Deutz, N.E., Honig, A., 2002. Cognition following acute tryptophan depletion: difference between firstdegree relatives of bipolar disorder patients and matched healthy control volunteers. Psychol. Med. 32 (3), 503-515.

Squire, L.R., 1987. The organization and neural substrates of human memory. Int. J. Neurol. 21-22, 218-222.

Stahl, S.M., 2008. Essential Psychopharmacology: Neuroscientific Basis and Clinical Applications. Cambridge University Press, New York.

Stancampiano, R., Cocco, S., Cugusi, C., Sarais, L., Fadda, F., 1999. Serotonin and acetylcholine release response in the rat hippocampus during a spatial memory task. Neuroscience 89 (4), 1135-1143.

Steckler, T., Drinkenburg, W.H., Sahgal, A., Aggleton, J.P., 1998a. Recognition memory in rats-II. Neuroanatomical substrates. Prog. Neurobiol. 54 (3), 313-332.

Steckler, T., Sahgal, A., Aggleton, J.P., Drinkenburg, W.H., 1998b. Recognition memory in rats-III. Neurochemical substrates. Prog. Neurobiol. 54 (3), 333-348.

Stein, D.J., Stahl, S., 2000. Serotonin and anxiety: current models. Int. Clin. Psychopharmacol. 15 (Suppl. 2), S1-S6

Stewart, M.E., Deary, I.J., Ebmeier, K.P., 2002. Neuroticism as a predictor of mood change: the effects of tryptophan depletion. Br. J. Psychiatry 181, 242-247.

Talbot, P.S., Watson, D.R., Barrett, S.L., Cooper, S.J., 2006. Rapid tryptophan depletion improves decision-making cognition in healthy humans without affecting reversal learning or set shifting. Neuropsychopharmacology 31 (7), 1519-1525.

Taylor, S.F., Kornblum, S., Lauber, E.J., Minoshima, S., Koeppe, R.A., 1997. Isolation of specific interference processing in the Stroop task: PET activation studies. Neuroimage 6 (2), 81-92.

Tork, I., 1990. Anatomy of the serotonergic system. Ann. N. Y. Acad. Sci. 600, 9-34 Tulving, E., 1983. Elements of Episodic Memory. Clarendon Press, New York.

Tulving, E., 1992. Memory systems and the brain. Clin. Neuropharmacol. 15 (Suppl. 1. Pt A), 327A-328A.

Van der Does, A.J., 2001. The effects of tryptophan depletion on mood and psychiatric symptoms. J. Affect Disord. 64 (2-3), 107-119.

van der Veen, F.M., Evers, E.A., Deutz, N.E., Schmitt, J.A., 2007. Effects of acute tryptophan depletion on mood and facial emotion perception related brain activation and performance in healthy women with and without a family history of depression. Neuropsychopharmacology 32 (1), 216-224.

van der Veen, F.M., Evers, E.A, van Deursen, J.A., Deutz, N.E., Backes, W. H. Schmitt, J.A., 2006. Acute tryptophan depletion reduces activation in the right hippocampus during encoding in an episodic memory task. Neuroimage 31 (3), 11881196.

Vizi, E.S., Kiss, J.P., 1998. Neurochemistry and pharmacology of the major hippocampal transmitter systems: synaptic and nonsynaptic interactions. Hippocampus 8 (6), 566-607.

Walderhaug, E., Landro, N.I. Magnusson, A. 2008. A synergic effect between lowered serotonin and novel situations on impulsivity measured by CPT. J. Clin. Exp. Neuropsychol. 30 (2), 204-211.

Walderhaug, E., Lunde, H., Nordvik, J.E., Landro, N.I., Refsum, H., Magnusson, A. 2002. Lowering of serotonin by rapid tryptophan depletion increases impulsiveness in normal individuals. Psychopharmacology (Berl) 164 (4), 385-391.

Weltzin, T.E., Fernstrom, J.D., McConaha, C., Kaye, W.H., 1994. Acute tryptophan depletion in bulimia: effects on large neutral amino acids. Biol. Psychiatry 35 (6), 388-397.

Weltzin, T.E., Fernstrom, M.H., Fernstrom, J.D., Neuberger, S.K., Kaye, W.H., 1995. Acute tryptophan depletion and increased food intake and irritability in bulimia nervosa. Am. J. Psychiatry 152 (11), 1668-1671. 
Williams, J.H., Perrett, D.I., Waiter, G.D., Pechey, S., 2007. Differential effects of tryptophan depletion on emotion processing according to face direction. Soc. Cogn. Affect Neurosci. 2 (4), 264-273.

Williams, W.A., Shoaf, S.E., Hommer, D., Rawlings, R., Linnoila, M., 1999. Effects of acute tryptophan depletion on plasma and cerebrospinal fluid tryptophan and 5-hydroxyindoleacetic acid in normal volunteers. J. Neurochem. 72 (4), $1641-1647$.
Young, S.N., Ervin, F.R., Pihl, R.O., Finn, P., 1989. Biochemical aspects of tryptophan depletion in primates. Psychopharmacology (Berl) 98 (4), 508-511.

Young, S.N., Smith, S.E., Pihl, R.O., Ervin, F.R., 1985. Tryptophan depletion causes a rapid lowering of mood in normal males. Psychopharmacology (Berl) 87 (2), 173-177.

Zohar, J., Kennedy, J.L., Hollander, E., Koran, L.M., 2004. Serotonin-1D hypothesis of obsessive-compulsive disorder: an update. J. Clin. Psychiatry 65 (Suppl. 14), $18-21$ 